\title{
Optical Properties of Spherical Colloidal Nanocrystals
}

\author{
Giovanni Morello \\ ${ }^{1}$ Nanoscience Institute of CNR, National Nanotechnology Laboratory (NNL), \\ ${ }^{2}$ Center for Biomolecular Nanotechnologies @UNILE, IIT, Arnesano (LE), \\ Italy
}

\section{Introduction}

The desire to fabricate materials with novel or improved properties is a powerful stimulus for the development of materials science. Thermal and electrical conduction, optical response, energy conversion and storage are just a few of the large number of properties underwent a very fast evolution in the last decade thank to the birth of a new branch of materials science and technology defined "Nanotechnology". Nanotechnology includes the totality of the physical, chemical, biological and engineering knowledge involving artificial structures whose properties are controlled at the nanometer level. Among the multitude of nanomaterials created, a particular class of them is becoming very popular and represents nowadays the most fascinating and potentially revolutionary inorganic semiconductor structure, which is the family of the colloidal quantum dots. They are often referred to as "nanocrystals" and the colloidal definition reveal their chemical origin. Actually, the chemical synthesis currently represents the most effective way to obtain high quality (in terms of size control, narrow size distribution, good crystalline structure and high optical performances) nano-objects on a gram scale which can be handled as ordinary chemical substances and implemented in several opto-electronic devices as well as biological ambient. Today, colloidal nanocrystals are successfully used as active media in lasers (Chan, et al., 2004; Klimov et al., 2000, 2007), LEDs (Anikeeva et al., 2009; Caruge et al., 2008), photovoltaic (Gur et al., 2005; Huynh et al., 2002; Kim et al., 2003), sensors (Oertel et al., 2005), biological labelling (Deka et al., 2009; Michalet et al., 2001), photo catalysis (HewaKasakarage et al., 2010). Chemical syntheses allow for the fabrication of nanocrystals (NCs) with nearly atomic precision. They are currently prepared in a variety of compositions as nearly spherical particles (Peng et al., 2000), elongated nanorods (Krahne et al., 2011), and other more complex structures like tetrapods (Fiore et al., 2009) and octapods (Miszta et al., 2011; Zhang et al., 2011). Moreover, the sophistication with which inorganic nanoparticles can be prepared has inspired the creation of multi-combined nano-systems having different compositions ranging from the all-semiconductor to hybrid semiconductor-metal nanoparticles possessing on demand properties in terms of electronic levels energy. As a consequence, the in-depth knowledge of the electronic structure of nanocrystals became of fundamental importance and a huge effort on the theoretical and experimental point of view has been made in the two last decades dedicated to their study and comprehension. 
This chapter wants to provide an overview of the main studies carried out on spherical nanocrystals on what concerns their electronic structure and optical properties. Particular attention will be devoted to the review of the role played by defect states (especially surface states) on the final optical performances by means of steady-state and time-resolved spectroscopy. For the sake of clarity, the reported discussions will concern two main kinds of nanocrystals, namely CdTe and CdSe. They represent the ideal cases study, since these two materials have historically been the most studied in the field of semiconductor nanostructures. The reason lies on the fact that they cover great part of the optical properties possessed by a number of different other nanocrystals and moreover they present the two main crystallographic symmetries, namely cubic (CdTe) and wurtzite (CdSe).

The chapter is organized as follows: Section 2 will be dedicated to the basic concepts of the low-dimensional systems. The idea is to provide a comprehensive overview of the physics at the basis of the systems studied. We will start from the most common problem consisting in solving the 1-D Schrödinger equation for an electron in a box, extending the discussion to the types of confinement (1-D, 2-D, 3-D) a nano-object undergoes. The size dependence will be reviewed, by distinguishing the different degrees of confinement. A final part will be devoted to the study of the electronic structure of spherical nanocrystals. Section 3 will provide an extensive discussion about the most important recombination processes occurring in spherical nanocrystals. These processes will be basically separated in two big families: radiative and non radiative processes. In the first case we will deal with relaxation processes involving the emission of one or more photons due to electron-hole annihilation. In the second case the excess energy is released as heat inside the materials and/or by excitation of new electron-hole pairs by Auger-like processes. Since great part of the potential applications of colloidal nanocrystals concerns with light emitting devices, particular emphasis is dedicated to the non radiative pathways limiting their optical performances. A review of the main studies reported in literature will be presented, with particular attention to the parameters affecting them, which are the size, the temperature, the excitation density and the surface quality. About the latter, the impact of surface states on the optical properties of CdSe NCs will be treated by means of a four-level model. A last section is dedicated to the Conclusions.

\section{Fundamentals of nano-physics}

This first section wants to stress the point that any development in nanoscience necessarily requires an understanding of the physical laws governing the matter at the nanoscale and of how the interplay of the various physical properties of a nanoscopic system translates into some novel behaviour or into a new physical property. In this sense, the section will be an overview of the basic physical laws that govern the nanomaterials, with particular emphasis on quantum dots, being the subject of the chapter.

\subsection{Quantum confinement on low dimensional systems}

The phenomenon of the quantum confinement (i. e. the size quantization) can be observed in systems where the motion of electrons or other particles (holes, excitons, etc.) is restricted at least in one dimension by some potential energy profile. Such a system is usually referred to as a "low dimensional" system. The energy spectra and the wavefunctions localization 
depend on the type of restriction in one, two or three dimensions, as well as on the size of the nanostructure. In particular, the quantum phenomena start to be noticeable when the lateral extension of the potential well becomes comparable to the particle wavelength. In order to better understand this concept, let us consider the case of the electrons. As elementary particles they exhibit the wave-particle duality of the matter following the "de Broglie" relation (de Broglie, 1924,1925). When immersed in a solid the electron is treated as a particle having an effective mass $\mathrm{m}^{*}$ accounting for the periodicity of the crystal potential. Its linear momentum $\mathrm{p}$ can be written in terms of its wave-like nature, $\mathrm{p}=\hbar \mathrm{k}$, where $\hbar$ is the Dirac's constant (the Planck's constant divided by $2 \pi$ ) and $k$ represents its wavenumber, associated to the de Broglie wavelength $\lambda=2 \pi / k$. Electrons in a bulk solid are treated as particles not feeling the borders by imposing the periodic boundary conditions, so that the wavefunctions and the energies are not affected by the real spatial extension of the solid. When the solid dimensions approach the electron wavelength, the permitted wavefunctions and energies undergo a series of restrictions in terms of continuity and absolute values. In few words, the system starts to be considered as "quantized". The simplest example of quantum confinement is an electron enclosed in a one-dimensional quantum box having lateral size a and infinitely high walls. In figure 1 we can see the situation in terms of energy and wavefunctions.

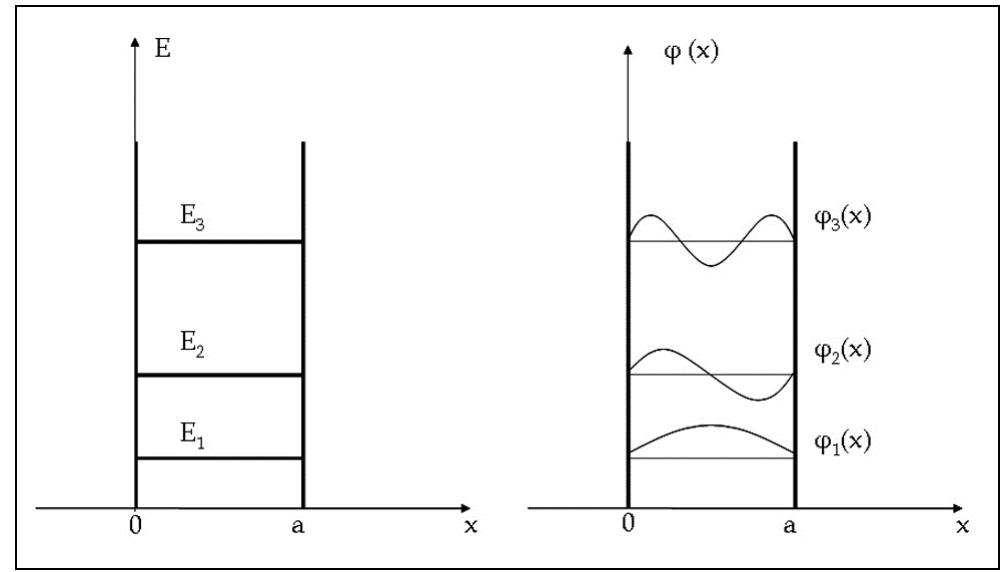

Fig. 1. Electron in a potential well. Energy levels and wavefunctions.

The problem constitutes a classical textbook case that can be approached by solving the Schrödinger equation in one dimension (Yoffe, 2001):

$$
\frac{d^{2} \varphi}{d x^{2}}+\frac{2 m e}{b^{2}}=V
$$

with $V=0,0<x<a$ and $V=\infty, x \leq 0, x \geq a$. The solutions of this second-order linear differential equation are in the form:

$$
\varphi(x)=A \cdot e^{i k x}+B \cdot e^{-i k x} .
$$


For an infinitely high potential barrier, the electron wavefunction must vanish at the borders of the potential well, such as $\varphi(0)=0$ and $\varphi(a)=0$. The condition $\varphi(0)=0$ produces $B=-A$, such that:

$$
\varphi(x)=A\left(e^{i k x}-e^{-i k x}\right)=2 i A \sin (k x) .
$$

Substituting $\varphi(x)$ in the Schrödinger equation one can obtain the following expression for the electron energy:

$$
E=\frac{\hbar^{2} k^{2}}{2 m} .
$$

The condition $\varphi(\mathrm{a})=0$ produces the following identity:

$$
\varphi(a)=2 i A \sin (k a)=0 .
$$

The identity is verified if $\mathrm{ka}=\mathrm{n} \Pi$, with $n=1,2,3, \ldots$ The parameter $\mathrm{k}$, therefore, results quantized, and the separation between two consecutive allowed values is $\Delta \mathrm{k}=\Pi / \mathrm{a}$. In terms of energy:

$$
E=\frac{\hbar^{2} k^{2}}{2 m}=\frac{\hbar^{2} \pi^{2} n^{2}}{2 m a^{2}} .
$$

The lowest energy for the electron, called Ground State energy, is obtained for $\mathrm{n}=1$,

$$
E=\frac{\hbar^{2} \pi^{2}}{2 m a^{2}} .
$$

The expression above defines the minimum energy possessed by a quantized system and is termed "point-zero energy" and constitutes a fascinating manifestation of the Heisenberg Uncertainty Principle. In fact, from

$$
\Delta x \cdot \Delta p \geq \frac{\hbar}{2}
$$

follows

$$
\Delta p \geq \frac{\hbar}{2 \Delta x}=\frac{\hbar}{a}
$$

since a represents the potential well dimension and $\Delta x \leq a / 2$. In terms of averaged kinetic energy:

$$
\left\langle E_{k}\right\rangle=\left\langle\frac{p^{2}}{2 m}\right\rangle \geq \frac{(\Delta p)^{2}}{2 m} \Rightarrow\left\langle E_{k}\right\rangle \geq \frac{\hbar^{2}}{2 m a^{2}} .
$$

This expression gives the theoretical lower limit of the possible kinetic energy value for a quantum particle confined in a box (one-dimensional, in this case) having size a. In the reality we find an actual value about $\Pi^{2} \approx 10$ times larger. 
The role of the system size on the confinement energy is played by the $1 / \mathrm{a}^{2}$ factor according to which larger the size a, smaller both the point-zero energy and the spacing in the k-space. This leads to smaller absolute energy spacing, whereas the relative spacing is expressed by

$$
E_{n+1}-E_{n}=\frac{\hbar^{2} \pi^{2}}{2 m a^{2}}\left[(n+1)^{2}-n^{2}\right]=\frac{\hbar^{2} \pi^{2}}{2 m a^{2}}(2 n+1),
$$

and increases with increasing $\mathrm{n}$.

The wavefunctions look like the standing waves on a string. Figure 1 plots the energies of the allowed states and the relative wavefunctions. They have increasing number of nodes with increasing energy according to the fact that more nodes mean shorter wavelength and higher momentum (i.e., energy).

\subsection{Classification of quantum confined systems}

In general, all the quantum confined systems can be classified on the basis of the number of dimensions along which the motion of electrons is coerced. The usual terminology refers to as 1-, 2-, 3-D confinement, in which the more evident effect consists in the modulation of the density of states function and a restriction of the allowed energies. In order to better understand what happens to a system when its dimensions start to shrink, one needs to begin from a non-confined structure, such as a bulk material. Here, one can assume that $\mathrm{N}$ electrons are not bound to individual atoms such that they can be considered "free" to move in three directions. If we suppose that, as a first approximation, the electron-electron interactions and the crystal potential are negligible (free electron gas model) (Pines, 1963) we can write the kinetic energy of an electron moving in the solid with velocity $\vec{v}=\left(v_{x}, v_{y}, v_{z}\right)$ :

$$
E=\frac{1}{2} m \vec{v}^{2}=\frac{1}{2} m\left(v_{x}^{2}+v_{y}^{2}+v_{z}^{2}\right) .
$$

The corresponding wavevector is derived from the relation

$$
\vec{p}=m \vec{v}=\hbar \vec{k}
$$

and the corresponding wavelength $\lambda=2 \Pi /|\mathrm{k}|$.

On the wavefunction point of view the condition of infinite solid is expressed by imposing the so called boundary conditions. They consist in the continuity of the wavefunction $\varphi(x, y, z)$ at the border of the real finite solid of dimensions $d_{x}, d_{y}, d_{z}$ :

$$
\left\{\begin{array}{l}
\varphi(x, y, z)=\varphi\left(x+d_{x}, y, z\right) \\
\varphi(x, y, z)=\varphi\left(x, y+d_{y}, z\right) \\
\varphi(x, y, z)=\varphi\left(x, y, z+d_{z}\right)
\end{array} .\right.
$$

The solution of the 3-D Schrödinger equation gives a factored function, written as the product of three independent functions:

$$
\varphi(x, y, z)=\varphi(x) \varphi(y) \varphi(z)=A \exp \left(i k_{x} x\right) \exp \left(i k_{y} y\right) \exp \left(i k_{z} z\right)
$$


In the argument of the exponential functions, $k_{x, y, z}$ is such that $\pm \Delta k_{x, y, z}= \pm n 2 \Pi / d_{x, y, z}$ with $n$ integer. In a bulk (having $d$ much larger than the electronic wavefunction), this condition tells us that all the values of allowed $\mathrm{k}$ are contained in a sphere in the k-space with a quasicontinuous distribution of states. At this point, it is useful to introduce the concept of Density of States (DOS) function $\mathrm{D}_{3 \mathrm{~d}}(\mathrm{k})$, intended as the number of states for unitary interval of wavenumbers. The electrons in a solid having a wavenumber $\mathrm{k}$ included in the interval between $\mathrm{k}$ and $\mathrm{k}+\Delta \mathrm{k}$ belong to the function $\mathrm{D}_{3 \mathrm{~d}}(\mathrm{k}) \Delta \mathrm{k}$. The total number of electrons contained in the sphere (having a maximum wavenumber $k_{\max }$ ) is

$$
N=\int_{0}^{k_{\max }} D_{3 d}(k) d k
$$

Since the volume of the sphere is proportional to $\mathrm{k}^{3}$ the number $\Delta \mathrm{N}(\mathrm{k})$ of electrons inside the interval $\mathrm{k}+\Delta \mathrm{k}$ is proportional to $\mathrm{k}^{2} \Delta \mathrm{k}$. Therefore

$$
D_{3 d}(k)=\frac{d N(k)}{d k} \propto k^{2}
$$

Now, it is possible to give the expression of the number of states in a unitary interval of energy $D_{3 d}(E)$. Since $E(k) \propto k^{2}$, thus $k \propto \sqrt{E}, d k / d E \propto 1 / \sqrt{E}$. It follows

$$
D_{3 d}(E)=\frac{d N(E)}{d E}=\frac{d N(k)}{d k} \frac{d k}{d E} \propto E / \sqrt{E}=\sqrt{E}
$$

In figure 2 we can see the situation in terms of Energy and Density of States.

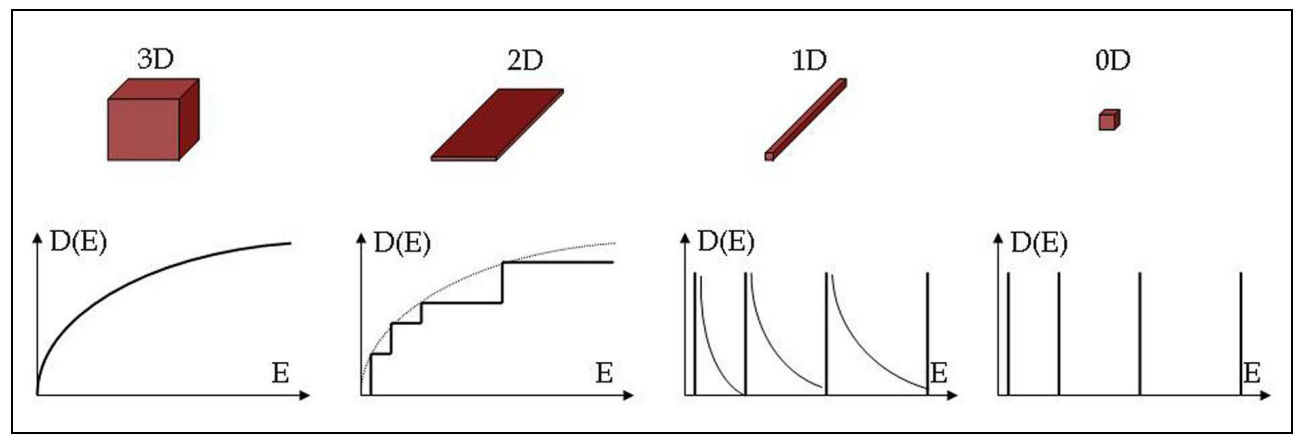

Fig. 2. Evolution of the "Density of States" (DOS) function by varying the degree of confinement. Reprinted from Physics Reports, 501, Krahne, R.; Morello, G.; Figuerola, A.; George, C.; Deka, S. \& Manna, L., Physical properties of elongated inorganic nanoparticles, 75-221, Copyright (2012), with permission from Elsevier.

The next step is to consider a system in which just a spatial dimension is reduced down to few nanometres, so that the electrons can freely move on a plan giving rise to the so called "2-D electron gas" (Davies, 1998). The quantization acts in the shrunk dimension (let us say the direction $\vec{z}$ ) along which the wavenumber assumes discrete values, $k_{z}=n_{z} \Delta k_{z}$ where 
$\Delta \mathrm{k}_{\mathrm{z}}=\Pi / \mathrm{d}_{\mathrm{z}}\left(\mathrm{d}_{\mathrm{z}}\right.$ being the size in the $\overrightarrow{\mathrm{z}}$ direction). On the Density of States point of view, we can consider a continuous distribution in the $x-y$ plane and an ensemble of infinite states equidistant one to each other. The number of states with $\mathrm{k}$ in the interval $\mathrm{k} \div \mathrm{k}+\Delta \mathrm{k}$ is now proportional to $\mathrm{k} \cdot \Delta \mathrm{k}$ and the consequent DOS function

$$
D_{2 d}(k)=\frac{d N(k)}{d k} \propto k
$$

In terms of energy:

$$
D_{2 d}(E)=\frac{d N(E)}{d E}=\frac{d N(k)}{d k} \frac{d k}{d E} \propto \sqrt{E} / \sqrt{E} \propto C .
$$

This expression means that in the motion plane the density of the states is constant. Obviously, this holds for a fixed value of $\mathrm{k}_{\mathrm{z}}$. By changing $\mathrm{k}_{\mathrm{z}}$ results in an increasing of the density $\mathrm{D}_{2 \mathrm{~d}}(\mathrm{E})$ which assumes now a step-like function.

In a one-dimensional system another direction of confinement is introduced making the structure resembling to a long wire. In the long dimension the DOS function becomes

$$
D_{1 d}(k)=\frac{d N(k)}{d k} \propto C
$$

from which

$$
D_{1 d}(E)=\frac{d N(E)}{d E}=\frac{d N(k)}{d k} \frac{d k}{d E} \propto 1 / \sqrt{E} .
$$

As in the 2-D case, an energy quantization is present and the function $\mathrm{D}_{1 \mathrm{~d}}(\mathrm{E})$ follows a sawtooth-like function. In particular, it is characterized by a quasi-continuous distribution of states (the hyperbolas in figure 2), but delimited by a series of singularities.

When the carriers are confined in all the three spatial dimensions the system undergoes a triple quantization. As a consequence the wavenumbers of the allowed states $\left(k_{x}, k_{y}, k_{z}\right)$ are discrete and can be represented by a point in the k-space. In terms of energy, the Density of States collapses into a series of discrete values assuming a Dirac's Delta function, as shown in figure 2, resembling to an atomic-like distribution.

On the optical point of view, the main quantity considered in studying the properties of semiconductor materials (either bulk or confined systems) is their energy gap. It represents the energy spacing between the last occupied and the first unoccupied electronic state at the ideal temperature of $0 \mathrm{~K}$ and is defined as

$$
E_{g}=E_{0}+E_{q}+E_{C} .
$$

Here, $\mathrm{E}_{0}$ represents the energy gap at $0 \mathrm{~K}$ of the bulk semiconductor, $\mathrm{E}_{\mathrm{q}}$ is the contribution of the quantization effect and $E_{C}$ accounts for the Coulomb interaction between the e-h pairs present in the semiconductor. The relative contribution of the last two terms determines the degree of confinement that a system undergoes. To this purpose, it is useful to introduce the 
fundamental concept of the "exciton". An exciton is a bound system composed of an electron and a hole experiencing a mutual Coulomb interaction. An important parameter of the exciton is the so called Bohr radius. It is a characteristic quantity of each bulk material and represents the natural extension of the e-h pair when the carriers are free to orbit one around to each other. A parameter linked to the exciton and to its Bohr radius $\mathrm{a}_{\mathrm{B}}$ is the binding energy of the exciton $E_{b}$ :

$$
E_{b}=\frac{\hbar^{2}}{2 \mu a_{B}^{2}} .
$$

where $\mu$ is the reduced mass of the exciton, defined as $\frac{1}{\mu}=\frac{1}{\mathrm{~m}_{\mathrm{e}}}+\frac{1}{\mathrm{~m}_{\mathrm{h}}}, \mathrm{m}_{\mathrm{e}}$ and $\mathrm{m}_{\mathrm{h}}$ being the effective masses of electron and hole, respectively. This energy assumes always negative values (being a binding energy) and represents the energy needed to ionize the exciton, that is to sustain the electron and the hole as separate entities. The Bohr radius can be expressed in terms of some physical parameters, such as the dielectric constant of the semiconductor $\varepsilon$, the reduced mass of the exciton $\mu$ and the electron charge e:

$$
a_{B}=\frac{\hbar^{2} \varepsilon}{\mu e^{2}} .
$$

Such quantity is different for each material and particle (i.e. electrons, holes, excitons, etc.) and ranges from a few $\mathrm{nm}$ to some tens of $\mathrm{nm}$. Since the confinement acts as a constrainer for the e-h wavefunction extension, we can say that each material undergoes proper quantization effects, depending on its size and Bohr radius. In few words, the degree of confinement of a system expresses a measure of how much the real dimensions of the material affect the motion and the lifetime of an exciton in such material. Indeed, comparing the Bohr radius and the real size of a nanoparticle makes possible to define three different regimes of quantization: weak, intermediate and strong confinement. Let $a_{e}, a_{h}$ and $a_{e x}$ be the Bohr radii of electron, hole and exciton, respectively; if a is the size of the system considered (for instance the radius of a spherical nanoparticle), when a is smaller than $a_{e}, a_{h}$ and $\mathrm{a}_{\mathrm{ex}}$ the system is in the strong confinement regime since both the electron and the hole strongly feel the boundary of the nanocrystal. In the weak confinement regime, the nanocrystal size is larger than the electron and hole Bohr radius but smaller than the exciton Bohr radius. If a falls in between $a_{e}$ and $a_{h}$ then the nanoparticle experiences the intermediate confinement effect. It is important to note, this point, that the degree of confinement featuring a semiconductor nanocrystal depends on the particular material constituting itself, since all the playing quantities are characteristic of each component. Therefore, nanocrystals of different materials but having the same size undergo a different degree of confinement. As a few examples we can consider three materials: $\mathrm{CuCl}, \mathrm{CdSe}$, InAs with radius of about $6 \mathrm{~nm}$; their Bohr radii are $0.68 \mathrm{~nm}$ (Ohmura \& Nakamura, 1999), $5.7 \mathrm{~nm}$ (Millo et el., 2004) and $34 \mathrm{~nm}$ (Kong et al., 2006), respectively. As a consequence, the effect of the quantum confinement effect is different in each nanocrystal: $\mathrm{CuCl}$ experiences a weak confinement, CdSe an intermediate one and InAs undergoes a strong confinement regime. 


\subsection{Exciton fine structure of wurtzite $\mathrm{CdSe}$ and cubic CdTe nanocrystals}

CdSe and CdTe nanocrystals probably represent the most investigated nanostructures, for what concerns both their optical and their electronic properties. About the electronic structure of the lowest energy state, we will highlight some of the pioneering works which have represented important milestones in the field, namely the theoretical and experimental studies of Efros et al. (Efros et al., 1996). The concepts discussed here can be considered as of general validity for nanocrystals having hexagonal or cubic crystallographic structure and are applicable to nanocrystals of a wide range of dimensions.

In our discussion, we consider a nanocrystal with dimensions that are much larger than its lattice constant, such that the effective mass approximation (Efros et al., 1996) is applicable. This condition is practically fulfilled in all cases, since the nanocrystal diameter is hardly smaller than 2-3 nm. The notation used to name the quantum states of a nanocrystal closely follows that of an atomic system, consistently to the energy expression previously determined. Therefore, we define the total angular momentum $J=(L+S)$ as the sum of the total orbital angular momentum $\mathrm{L}$ and the multiplicity term $S$, i.e. the electron spin, and the relative momentum projections: $\mathrm{j}, \mathrm{l}$, and $\mathrm{s}$. The electron ground state has s-symmetry and presents a double degeneracy, which is due exclusively to the spin momentum. Thus $\mathrm{J}=0+1 / 2$, its projections are $\mathrm{j}= \pm 1 / 2$, and the state is conventionally indicated as $1 \mathrm{~S}_{\mathrm{e}}$. On the other hand, the first hole level, having a p-symmetry, is fourfold degenerate, having $\mathrm{J}=1+1 / 2=3 / 2(\mathrm{j}=3 / 2,1 / 2,-1 / 2,-3 / 2)$, and is named $1 S_{3 / 2}$. The composition of the two ground states yields the eightfold degenerate exciton ground state $1 \mathrm{~S}_{3 / 2} 1 \mathrm{~S}_{\mathrm{e}}$.
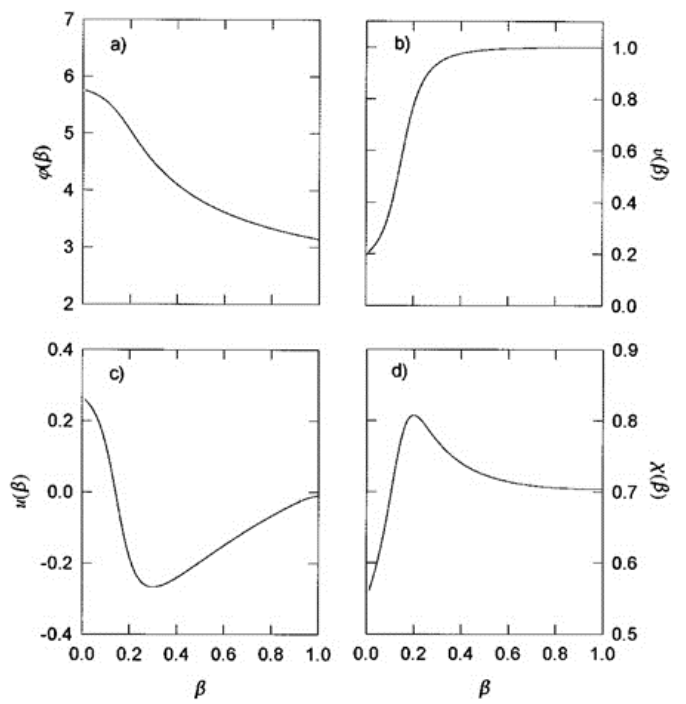

Fig. 3. (a) The dependence of the hole ground state function $\varphi(\beta)$ on the light to heavy hole effective mass ratio $\beta ;(b) v(\beta)$ associated with hole level splitting due to hexagonal lattice structure; $(c) \mathrm{u}(\beta)$ associated with hole level splitting due to crystal shape asymmetry; (d) $\chi(\beta)$ associated with exciton splitting due to the electron-hole exchange interaction.

Reprinted figure with permission from Efros, Al. L.; Rosen, M.; Kuno, M.; Nirmal, M.; Norris, D. J.\& Bawendi, M. Physical Review B, 54, 4843-4856, 1996. Copyright (2012) by the American Physical Society. 
The degeneracy of the ground state is lifted by various effects, such the internal crystal field, shape effects and the electron-hole exchange interaction. The first effect arises from an intrinsic property of semiconductors having hexagonal lattice structure (like CdSe) and therefore manifests itself in both bulk and nanoscale materials. The second effect takes into account the deviation from the ideal spherical shape of nanocrystals, while the third effect accounts for mixing of electron and hole spins. The first two effects can be grouped together, as they arise from the intrinsic asymmetry of the material/nanocrystal. The intrinsic crystal field produces a first splitting of the valence band, i.e. the lowest hole state, in the so called Kramers doublet, which consists of two doubly degenerate states with $j= \pm 1 / 2$ and $j= \pm 3 / 2$ (Efros et al., 1996). Let us define a parameter $\beta$ as the ratio between the mass of the light hole $\mathrm{m}_{\mathrm{lh}}$ and the mass of the heavy hole $\mathrm{m}_{\mathrm{hh}}$ (hence $\beta=\mathrm{m}_{\mathrm{lh}} / \mathrm{m}_{\mathrm{hh}}$ ). The energetic splitting due to the intrinsic crystal field is then expressed as (Efros et al., 1996):

$$
\Delta_{1}=\Delta_{C F} v(\beta)
$$

where $\Delta_{C F}$ is a parameter related to the crystal field (CF) splitting in a crystal with hexagonal structure, contributing to determine the hole ground state with $|j|=3 / 2$, while $v(\beta)$ is a function which is unique for each material (see (Efros et al., 1996) for details). It is worth to stress that $\Delta_{1}$ does not depend on the size of the nanocrystal. Moreover, since $v(\beta)$ is always positive (see figure $3 \mathrm{~b}$ ), the lowest hole level is fixed with the heavy hole state with $|j|=3 / 2$. In order to take the shape anisotropy into account, we can model a nanocrystal as an axially symmetric ellipsoidal particle (i.e. an ellipsoid with principal axes $a=b<c$ ), and define the ratio of the major to minor axes as $c / b=1+\varepsilon, \varepsilon$ being the ellipticity. The induced splitting in this case is:

$$
\Delta_{2}=2 u(\beta) E_{3 / 2}(\beta) \varepsilon
$$

Here, $u(\beta)$ is a dimensionless function associated with the hole level splitting due to the crystal shape (for details see (Efros et al., 1996), figure $3 c$ ) and $E_{3 / 2}(\beta)$ is the hole ground state energy which can be written as:

$$
E_{3 / 2}(\beta)=\frac{\hbar^{2} \varphi^{2}(\beta)}{2 m_{h h} a^{2}}
$$

where $\varphi^{2}(\beta)$ is a term related to the spherical Bessel functions and a is related to the nanocrystal size in the sense that for quasi-spherical nanocrystals $a=\left(b^{2} c\right)^{1 / 3}$.

Concerning $\Delta_{2}$, an important point is the trend in the function $\mathrm{u}(\beta)$, in particular for what concerns its sign. As shown in Figure $3 c, u(\beta)$ reverses its sign past a certain value of $\beta$, meaning that for some materials the shape anisotropy induces a negative splitting resulting in a possible inversion of the hole ground state between $|j|=3 / 2$ and $|j|=1 / 2$, since the global energy splitting is the sum of the single asymmetry contributions $\left(\Delta_{t}=\Delta_{1}+\Delta_{2}\right)$. A negative $\Delta_{2}$ is found, for example, in elongated CdSe nanorods, for which $\beta=0.28$, where a possible inversion would depend on the hole ground state energy and on the radius of rods (Krahne et al., 2011).

The exciton ground state results until now split in two fourfold degenerate excitonic states, having total angular momentum $\mathrm{F}=1$ and $\mathrm{F}=2$. The exchange interaction further contributes to an increase of the splitting of the remaining states, defining the fine structure for 
nanocrystals having a series of possible shapes, as depicted in figure 4 (Efros et al., 1996). The final configuration of the fine structure is based on the definition of the projection $f$ of the total angular momentum F. It assumes different values: one state with $f= \pm 2$, two states with $\mathrm{f}= \pm 1$ (named Upper and Lower, depending on the branch they originate) and two others with $\mathrm{f}=0$ (Upper and Lower). Three of them are optically active, namely the states $0 \mathrm{U}$, $\pm 1 \mathrm{U}$ and $\pm 1 \mathrm{~L}$, and the remaining \pm 2 and $0^{\mathrm{L}}$ states are passive. The \pm 2 state is optically forbidden because of the restrictions about the angular momentum conservation for photons (which cannot have an angular momentum \pm 2 , for example). The $0^{\mathrm{L}}$ has zero optical transition probability because of an interference phenomenon between the two indistinguishable states with zero angular momentum (Efros \& Rosen, 2000), due to the influence of the electron-hole exchange interaction. The shape of the nanocrystal plays the significant role on defining which of the above states represents the exciton ground state. For perfectly spherical nanocrystals the \pm 2 is the ground state, whereas in prolate nanocrystals an inversion of the \pm 2 with the $0^{\mathrm{L}}$ state can occur, because the state \pm 2 originates from the hole state with $|j|=3 / 2$, whilst $0^{\mathrm{L}}$ arises from the state $|j|=1 / 2$. When the conditions for the sign change of $\Delta_{t}$ are met, the ground state is inverted. A natural generalization of this concept can be found in the electronic structure of elongated nanocrystals (also named Nanorods (Krahne et al., 2011)) which can be approximated by axially symmetric prolate ellipsoids with ellipticity $\varepsilon$ defined as $\varepsilon=\left(2 a_{B} / b\right)-1$ (here, the long axis c can be replaced by the Bohr radius), with $b$ being the ellipsoid diameter and $a_{B}$ the Bohr radius. In the case of strong lateral confinement $\left(b<2 a_{B}\right)$, the ellipsoids are subject to a possible inversion of the ground state between \pm 2 and $0^{\mathrm{L}}$. This can happen because $\Delta_{2}$ becomes increasingly important in the strong confinement regime, and at some point it would cause the light hole state with $j= \pm 1 / 2$ to become the hole ground state. The coupling with the electron state $1 S_{e}$ yields a fourfold degenerate state, with angular momentum 0 (two states) and \pm 1 . The hole state with $j= \pm 3 / 2$ yields the second doubly degenerate state with momentum \pm 1 and \pm 2 . In practice, the new lowest exciton level would be the state $0^{\mathrm{L}}$ and the exciton fine structure resembles that of figure $4 \mathrm{c}$. For what concerns nanocrystals having a cubic structure, we will refer to another case study represented by CdTe nanocrystals. Efros et al. (Efros et al., 1996) calculated the size dependence of the band-edge splitting, showed in figure 5 for different shapes. In spherical nanocrystals the e-h exchange interaction split the exciton ground state into two states. The state at lowest energy is fivefold degenerate, presents total angular momentum $\mathrm{F}=2$ and results dark. The higher energy state is threefold degenerate, has a total angular momentum $\mathrm{F}=1$ and is bright. Contrary to the wurtzite case, the crystal field does not act as splitter, and just the shape anisotropy contributes to determine the order of the excitonic states in the real system (Efros et al., 1996).

On the experimental point of view, the fine structure of CdSe nanocrystals has been investigated by a number of experiments, more than CdTe one which presents a lot of degeneracy. The main difficulty in studying the fine structure is given by the size distribution of a sample of colloidal nanocrystals leading to an inhomogeneous broadening of the optical spectra which hides the fine distribution of the states. On the other hand, single nanoparticle experiments can provide information only ideally, since several effects contribute to destroy the advantages of spectral narrowing, namely intermittent emission (blinking) (Schlegel et al., 2002), spectral diffusion (Empedocles \& Bawendi, 1999) and possible photodegradation (Wang et al., 2003). Fluorescence line narrowing and photoluminescence excitation (Norris et al., 1996) experiments are the most useful steadystate methods to access a subset of the nanocrystals ensemble, whereas the cross polarized, 

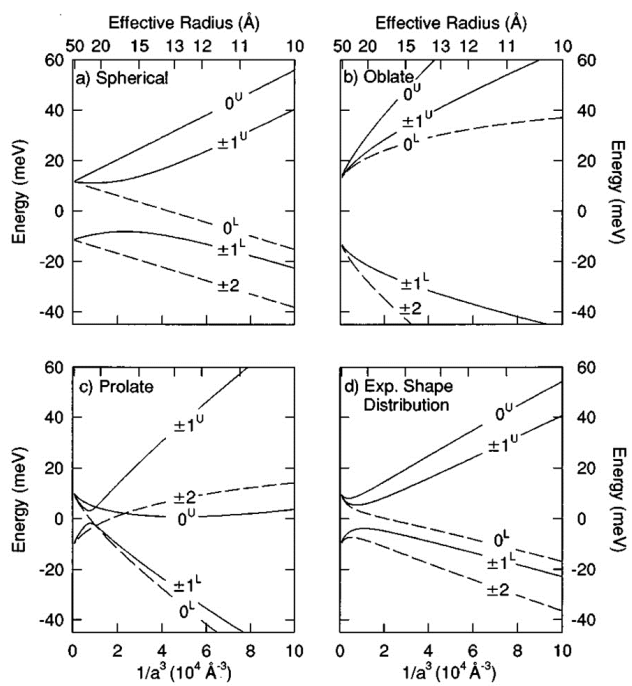

Fig. 4. Size dependence of the exciton ground state of CdSe NCs. (a) spherical NCs; (b) oblate NCs; (c) prolate NCs; (d) NCs having a size dependent ellipticity as determined from Efros et al., 1996. Solid/dashed lines indicate optically active/passive levels. Reprinted figure with permission from Efros, Al. L.; Rosen, M.; Kuno, M.; Nirmal, M.; Norris, D. J.\& Bawendi, M. Physical Review B, 54, 4843-4856, 1996. Copyright (2012) by the American Physical Society.

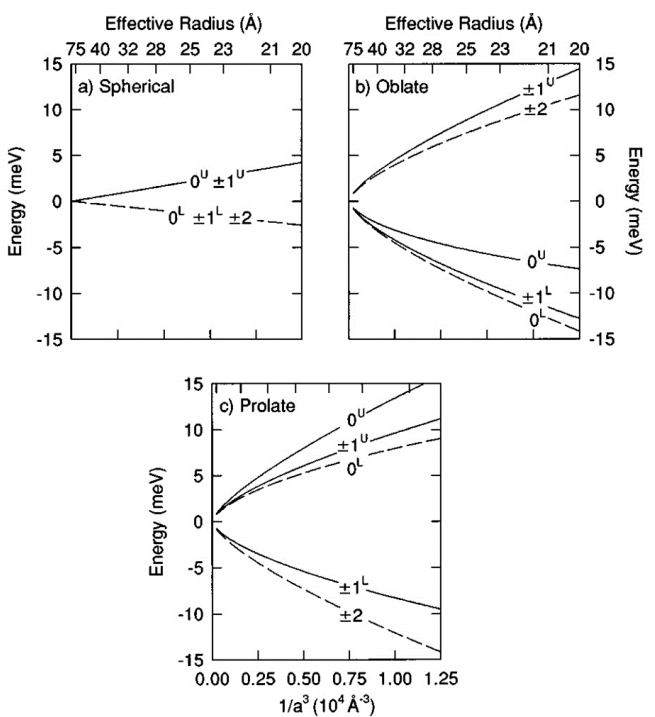

Fig. 5. The exciton fine structure of cubic CdTe NCs. (a) spherical NCs; (b) oblate NCs; (c) prolate dots NCs; Solid/dashed lines indicate optically active/passive levels. Reprinted figure with permission from Efros, Al. L.; Rosen, M.; Kuno, M.; Nirmal, M.; Norris, D. J.\& Bawendi, M. Physical Review B, 54, 4843-4856, 1996. Copyright (2012) by the American Physical Society. 
heterodyne detected third-order transient grating (CPH-3TG) method (Kim et al., 2009) has been employed to probe ultrafast transient dynamics in the fine structure distribution. On the luminescence point of view, the main difficulty is to be able to detect the very fast signal arising from the higher energy state of the fine structure. On this regard, a few time resolved PL experiments have been reported in the literature, showing emission from higher states on the picoseconds time scale (Morello et al., 2007a; Moreels et al., 2011) and only by using a streak camera. Also in these experiments the inhomogeneous broadening contributes to hide some spectral features and the situation is further complicated by both the possible exchange among the states due to the unavoidable asymmetric shape (Moreels et al., 2011) and the intermixing between intrinsic and surface states (Califano et al., 2005; Morello et al., 2007). All these effects participate to the definition of the actual ground state inside the fine structure. Indeed, the ideal case of perfectly spherical and pure nanocrystals is often replaced by the real situation in which slightly asymmetric and defected nanocrystals are investigated. Therefore, it is not rare to assist to the inversion between bright and dark states (it is the case of most nanorods systems (Krahne et al., 2011) and some not perfectly spherical NCs) or to the mixing between dark and surface states leading to the partial brightening of the same (Califano et al., 2005). The role of surface states in terms of quantum yield and lifetime will be treated in the last part of the chapter.

\section{Excitation and relaxation processes in colloidal nanocrystals}

After excitation of charge carriers, the system tends to restore the initial equilibrium state losing the excess energy. All the processes involved in this action constitute the whole of the relaxation processes of the system. Restoring of the initial equilibrium state can occur in several ways, and dealing with nanoparticles instead of bulk semiconductor introduces some complications that will be analysed in the following paragraphs. The most common energetic relaxation process is the heating of the material. It consists in the trigger of an emission of phonons (lattice vibrations), mediated by the electron-phonon interaction. This kind of interaction does not affect only the decay toward lower energy states (carrier cooling (Nozik, 2001)), but it can influence the promotion of charge carriers to higher states through the absorption of phonons inducing, in particular cases, the ejection of charges away from the material. This particular process, holding for both electrons and holes, is strongly dependent on the temperature and is often referred to as "thermal escape" (Valerini et al., 2005; Morello et al., 2007b). In the following we will give a complete description of thermal escape occurring in CdSe (Valerini et al., 2005) and CdTe nanocrystals (Morello et al., 2007b). Another phenomenon of electron-hole annihilation is due to carrier-carrier scattering, via the so called "Auger effect". It manifests when more than one e-h pair is present into the semiconductor and consists in the transfer of the energy owned by an e-h couple to a third particle (another electron, hole, or exciton). This relaxation pathway is strongly dependent on both the morphology of the semiconductor (shape and dimensions) and the level of charge carrier injection. The alternative way for exciton relaxation is the emission of photons that, in general, is less efficient than heating. The reason lies on the fact that the emission (likewise absorption) of photons is hindered by the selection rules of the optical transitions: the photons can involve states with angular momentum equal to $0, \pm 1$, whereas the branches of phonons are distributed on the whole first Brillouin's region, i.e. they possess all the possible electron momenta, thus allowing for connection of states having different angular momentum. This condition is particularly favourable in taking place of intraband relaxation by means of phonon emission instead of radiative emission. 
In a semiconductor, the absorption of a photon with energy larger than the energy gap, creates an e-h pair. Often, the energy of the exciting photon does not match perfectly the energy gap of the material. In particular, this is true in bulk for which the continuum of states allows for a continuous distribution of excited electrons in the conduction band. Such a distribution, at the thermal equilibrium, follows a Boltzmann's statistics depending on the temperature of the system, with electrons being within $\mathrm{k}_{\mathrm{B}} \mathrm{T}$ from the bottom of the conduction band and holes within $\mathrm{k}_{\mathrm{B}} \mathrm{T}$ from the top of the valence band (Nozik, 2001). Quantum confinement strongly affects carrier cooling efficiency by phonon scattering. In bulk semiconductors, exciton energy relaxation results mainly from cascade LO-phonon emissions, whereas interaction with acoustic phonons is less important. In nanocrystals smaller than $10 \mathrm{~nm}$, or in general when the discreteness of electron-hole states becomes essential, optical phonons cannot provide an efficient relaxation channel. The dispersion curve of optical phonons is nearly wavenumber-independent, and LO-phonon energies can only be weakly deviated from the bulk ones (Trallero-Giner et al., 1998). Therefore, the relaxation via multiple LO-phonon emission requires $\Delta \mathrm{E}_{1,2} \approx \mathrm{nE}_{\mathrm{LO}}$, where $\mathrm{n}$ is an integer number. Unlike in bulk crystals where there exists a continuous spectrum for quasiparticles, in nanocrystals there are not intermediate states between the two lowest excited ones. Therefore, the probability of the above process is very low because multiple phonon emission should occur via virtual intermediate states. The corresponding relaxation rate is lower than the typical electron-hole recombination rates. Thus, the relaxation between the excited states in nanocrystals is essentially inhibited. This effect is the so called "phonon bottleneck"(Gaponenko, 1998; Nozik, 2001). Phonon bottleneck in nanoparticles has been studied in the past years, and the results are controversial (Guyot-Sionnest et al., 1999; Klimov \& McBranch, 1998; Woggon et al., 1996). The excited carriers should have an infinite relaxation lifetime for the extreme, limiting condition of a phonon bottleneck; thus, the carrier lifetime would be determined by non radiative processes, and PL would be absent (Gaponenko, 1998). In the case that the relaxation times are not excessively long and PL is observed, the results are not indicative of a phonon bottleneck, although relatively long hot electron relaxation times (tens of picoseconds) compared with what is observed in bulk semiconductors are observed. Some researchers (Klimov, 2000) also found very fast electron relaxation dynamics on the order of $300 \mathrm{fs}$ from the first-excited 1P to the ground 1S state on II-VI CdSe colloidal NCs using interband pump-probe spectroscopy, which was attributed to an Auger process for electron relaxation that bypassed the phonon bottleneck. In this specific Auger process, the excess electron energy is rapidly transferred to the hole, which relaxes through its dense spectrum of states. The final equilibration step results in complete relaxation of the system. The electrons and holes can recombine, either radiatively or non radiatively, to produce the population densities that existed before photoexcitation.

A detailed study of the NCs photophysics with a particular attention to non radiative processes is not only interesting for fundamental physics, but it is also relevant to the exploitation of nanocrystals in practical applications. In this context we will deal with relaxation of carriers after they reach the lowest, potentially radiative, excited state. Again, the following discussion is based on the properties of CdSe and CdTe nanocrystals. Several relaxation processes have been proposed to explain the photophysics of CdSe QDs, including the thermally activated exciton transition from dark to bright states (Crooker et al., 2003) and carriers surface localization in trap states (Lee et al., 2005). Moreover, it has been shown that at room temperature the main non radiative process in CdSe/ZnS 
core/shell QDs is thermal escape, assisted by multiple longitudinal optical (LO) phonons absorption (Valerini et al., 2005), while at low temperature evidence for carrier trapping at surface defects was found. Despite these results, the role and the chemical origin (Wang et al., 2003b) of the surface defect states in the radiative and non radiative relaxation in nanocrystals has not been clarified completely. The existence of surface states due to unpassivated dangling bonds has been invoked to explain anomalous red-shifted emission bands in colloidal nanocrystals (Lee et al., 2005). On the contrary, it has been shown that above-gap trap states (Bawendi et al., 1992) affect the ultrafast relaxation dynamics (Cretì et al., 2005) and the single nanoparticle PL spectra of CdSe quantum rods (Rothenberg et al., 2005) due to charge trapping and local electric field fluctuations. These effects are expected to be dependent on the chemical composition of the QDs, on the density of surface defects and on the nanocrystals size. In what follows we will review the main processes affecting the light emission properties of nanocrystals, namely exciton-phonon coupling, Auger interactions and surface quality.

\subsection{Exciton-phonon interaction}

The main features that distinguish nanostructures from bulk materials originate from the localized character of the electron and hole wavefunction and the discrete nature of their optical transitions. In terms of carrier-phonon coupling, it essentially affects and defines the properties of homogeneous broadening of the PL signal. In the bulk, the broadening is mainly determined by the polar coupling of both electrons and holes to optical phonons (Liebler et al., 1985). The piezoelectric and deformation potential coupling of both carriers to acoustic phonons is usually not very important (Takagahara, 1996). This situation is different in nanostructures, where the local charge neutrality character of the exciton becomes predominant, producing an ideal null polar coupling of the exciton to optical phonons (Schmitt-Rink et al., 1987). This holds true for infinite barriers, such that the electron and hole wavefunctions are practically identical. In general, in real systems (finite barrier) just a decrease of the polar coupling with increasing barrier is expected (Muljarov \& Zimmerman, 2007; Nomura \& Kobayashi, 1992; Schmitt-Rink et al., 1987). On the other hand, since the deformation potential coupling is proportional to $1 / \mathrm{R}^{2}$ ( $\mathrm{R}$ being the radius of a spherical dot), the coupling strength to acoustic phonons is increased as the dimensions are reduced below the Bohr radius (Gindele et al., 2000; Takagahara, 1996). Also, the temperature affects each of these contributions. The temperature dependence of the spectral line width can be expressed as (Rudin et al., 1990):

$$
\Gamma(T)=\Gamma_{0}+\sigma T+\gamma N_{L O}(T)
$$

where $\Gamma_{0}$ is the inhomogeneous broadening, $\sigma$ is the exciton-acoustic phonons coupling coefficient, $\gamma$ is the coefficient accounting for the exciton-optical (LO) phonon coupling and $\mathrm{N}_{\mathrm{LO}}$ represents the Bose function for LO phonon occupation:

$$
N_{L O}=\frac{1}{e^{E_{L O} / k_{B} T}-1}
$$

In the expression above $E_{\mathrm{LO}}$ is the energy of the longitudinal optical phonon with momentum $\mathrm{k}=0$ (i.e. the phonon that preferably couples to the lowest exciton state) and $\mathrm{k}_{\mathrm{B}}$ 
is the Boltzmann's constant. Due to the different energetic dispersion curves of acoustic and optical phonons, the two couplings dominate at different temperature ranges. The acoustic phonons, having smaller energies ( $\mathrm{a}$ few $\mathrm{meV}$ ) heavily contribute to the broadening at low temperature (until 50-70 K), whereas the optical phonons (with energies of a few tens of $\mathrm{meV}$ ) dominate at higher temperature (Morello et al., 2007b). In a general fit procedure, the experimental data of the broadening are extracted by fitting the PL spectra to a convolution of a number of Gaussian peaks (usually not more than three) from which the broadening of the PL is extracted. In order to discern the inhomogeneous and homogeneous components the extracted values are fitted to eq. 29. In figure 6, it is shown the case of a set of three samples of CdTe NCs having different size whose temperature dependent broadenings have been analyzed by eq. 29 (Morello et al., 2007b).

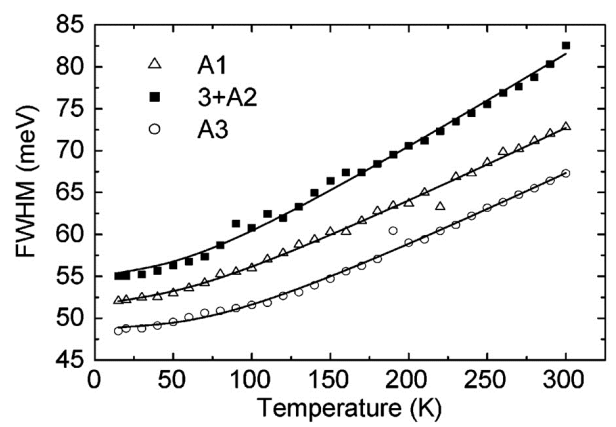

Fig. 6. PL broadening as a function of temperature (symbols) and relative best fit to eq. 29 (continuous lines) for three different size of CdTe NCs. Reprinted with permission from Morello, G.; De Giorgi, M.; Kudera, S.; Manna, L.; Cingolani, R. \& Anni, M., Temperature and size dependence of nonradiative relaxation and exciton-phonon coupling in colloidal CdTe quantum dots. J. Phys. Chem. C, 111, 5846-5849. Copyright (2012) American Chemical Society.

The three samples were called A1 (average diameter of $4.2 \mathrm{~nm})$, A2 (4.9 nm) and A3 (5.9 nm) and the relative PL was well fitted by one Gaussian peak. As expected from eqs. 29-30 the broadening increases with the temperature by virtue of an increasing probability of existence of (optical) phonons. The above mentioned analysis produced the expected sizedependent behavior of the coupling coefficients between excitons and acoustic/optical phonons. In particular, it was found a coupling to acoustic phonons about three orders of magnitude larger than in the bulk system and increasing $\sigma$ with decreasing the NC size, consistently with the theoretical prediction of a strong increase of the coupling to acoustic phonons in zero-dimensional systems (Valerini et al., 2005). The exciton-LO phonon coupling, on the contrary, showed a smaller value respect to the bulk counterpart and moreover it was reduced in the smallest NCs, accordingly to an expected ideally null polar coupling in zero-dimensional systems.

An important contribution of the exciton-phonon interaction to the emission properties consists in the influence on the temperature dependence of the PL quantum yield. As usually observed in colloidal nanocrystals, the PL intensity exhibits a considerable decrease with increasing the temperature. Starting from $10 \mathrm{~K}$, the decrease is moderate in the low temperature regime (let us say until $100 \mathrm{~K}$ ) becoming heavier at temperature higher than 
about $150 \mathrm{~K}$. In general, the temperature-induced quenching includes a number of processes that could be identified by analyzing the trend of the PL integrated area as recorded at the different temperatures. The plot of the intensity as a function of $1 / \mathrm{k}_{\mathrm{B}} \mathrm{T}$ (where $\mathrm{k}_{\mathrm{B}}$ is the Boltzmann constant and $\mathrm{T}$ is the temperature) on a semi-logarithmic scale allows for the determination of the activation energy of the thermal processes triggered at high temperature, by fitting the experimental data with an Arrhenius law, using the expression

$$
I(T)=\frac{I_{0}}{1+\sum_{i=1}^{n} a_{i} e^{\left(-E_{i} / k_{B} T\right)}} .
$$

Here, $\mathrm{n}$ is the number of thermal processes, $\mathrm{E}_{\mathrm{i}}$ represents the activation energy of the i-th process and $a_{i}$ is a fitting parameter accounting for the relative weight of each exponential term.

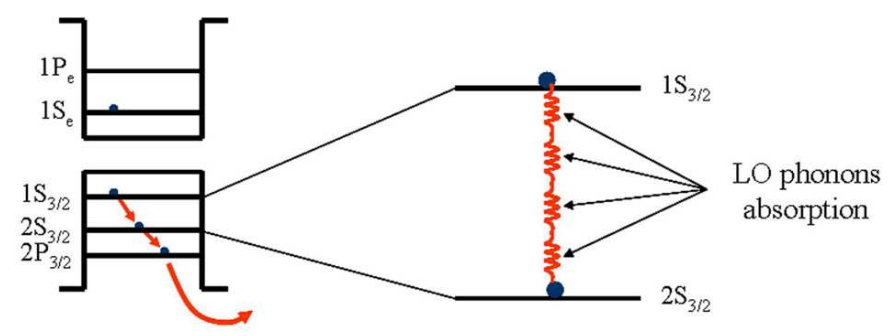

Fig. 7. Schematic description of thermal escape in CdTe NCs.

Among the thermal processes affecting the PL quantum efficiency of nanocrystals and involving exciton-phonon coupling we recall the "thermal escape". This process involves the carriers occupying the emitting state and consists in the absorption of a number of optical phonons such as to cover the energetic spacing between the emitting and the first, high energy, allowed state. Since the protagonist physical phenomenon is the absorption of phonons, the essential requirement to occur is the nonzero probability of existence of a certain number of optical phonons in the material (eq. 30). Figure 7 schematizes the mechanism for a CdTe NC: after excitation, electrons and holes undergo ultrafast intraband relaxation, until they occupy the lowest permitted energetic level before annihilation. This point, the e-h pair can recombine in several ways, among which we find the radiative emission, trapping, Auger effects and exciton-phonon coupling. If we consider a high degree of purity (neglecting trapping) and a low excitation density level (avoiding Auger processes) the photon emission must compete only with carrier-phonon interaction. When the latter has a high rate (at high temperature) the absorption of a number of optical phonons leads one or both the carriers (the hole, in the case of CdTe) to occupy the higher energy level. From such state the further absorption of phonons is facilitated by the reduced energetic spacing required to jump into the successive hole level, since a smaller number of phonons is needed. The process is iterated until the carrier is ejected from the nanocrystal. Therefore, the involved physical quantities are the temperature (according to eqs. 29-30) and the energetic spacing between the first two excited states (defining the maximum number of absorbed phonons) being the most energetically spaced. A good treatment of the thermal 
escape on the theoretical and experimental point of view has been provided by Valerini et al. (Valerini et al., 2005) and Morello et al. (Morello et al., 2007b).

We can consider the following rate equation for the carrier density n (Valerini et al., 2005):

$$
\frac{d n}{d t}=g(t)-\frac{n}{\tau_{\text {rad }}}-\frac{n}{\tau_{\text {act }}}-\frac{n}{\tau_{e s c}}
$$

where $g(t)$ is the generation term, $1 / \tau_{\text {rad }}$ is the radiative recombination rate, and $1 / \tau_{\text {esc }}$ is the thermal escape rate given by

$$
\frac{1}{\tau_{\text {esC }}}=\frac{1}{\tau_{0}}\left(e^{E_{L O} / k_{B} T}-1\right)^{-m}
$$

where $1 / \tau_{0}$ is a fitting parameter acting as a weight for the probability of carrier-LO-phonon scattering, and $\mathrm{m}$ is the number of LO phonons involved in the process. The rate of a generic thermally activated process is given by

$$
\frac{1}{\tau_{a c t}}=\frac{1}{\tau_{a}} e^{-E_{a} / k_{B} T},
$$

where $E_{a}$ is the activation energy and $1 / \tau_{a}$ is a fitting parameter acting as a weight for the probability of this process. The intensity of the PL emitted per unit time is given by

$$
I_{P L}(t)=\frac{n(t)}{\tau}=\frac{n_{0}}{\tau} e^{-t / \tau},
$$

where $\mathrm{n}_{0}$ is the initial carriers population and $\tau$ is the temperature-dependent PL decay time given by

$$
\frac{1}{\tau}=\frac{1}{\tau_{\text {rad }}}+\frac{1}{\tau_{\text {act }}}+\frac{1}{\tau_{\text {esc }}} .
$$

The integrated PL intensity is instead given by

$$
I_{P L}(T)=\int_{0}^{\infty} I_{P L}(t) d t=\frac{n_{0}}{1+\tau_{\text {rad }} / \tau_{\text {act }}+\tau_{\text {rad }} / \tau_{\text {esc }}} .
$$

Considering only the thermal escape the expression above becomes:

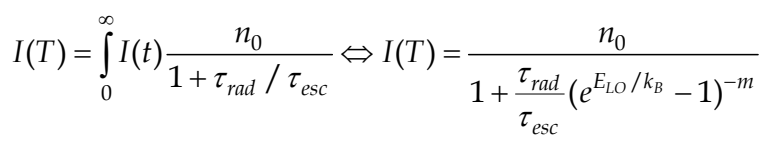

As a case study, we can consider a set of three samples of CdTe nanocrystals having different diameter, namely $4.2 \mathrm{~nm}, 4.9 \mathrm{~nm}$ and $5.9 \mathrm{~nm}$ (Morello et al., 2007b). From the absorption spectra it is possible to firstly deduce the energetic spacing among the lowest quantized states. In CdTe the two lowest absorption peaks arise from absorption of the 
lowest degenerate electron state $1 S_{e}$ and the two lowest hole states $1 S_{3 / 2}$ and $2 S_{3 / 2}$, as shown in figure 8 . Therefore, their energetic separation determines the jump to be executed by the charge carriers (in this case the holes) in order to escape from the nanocrystals. Due to the quantization effect, the first two states observed in absorption have the largest energy separation, thus they represent the major obstacle to the process. In the specific case of figure 8, the separations are 124.5, 96.5 and $82.2 \mathrm{meV}$ for samples A1, A2 and A3, respectively. By analysing the PL intensity vs. temperature with an Arrhenius function like eq. 38, it is possible to extract a number $\mathrm{m}$ of optical phonons involved in the quenching process, namely 5.6, 4.9 and 4 . Such number is not integer due to both the size dispersion of the samples and the statistic character of the physical quantity considered. By multiplying this number to the energy separation arising from the absorption spectra one obtains an energy value comparable to the separation between the two lowest excited states giving evidence for the occurred thermal escape process (see Morello et al., 2007b for details).

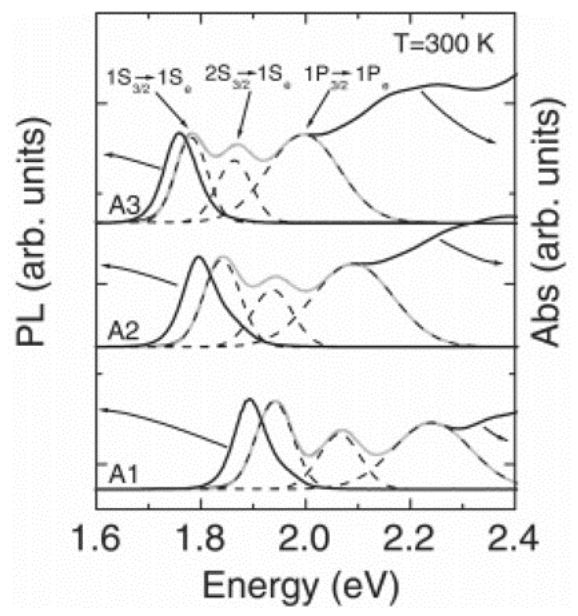

Fig. 8. Absorption and emission spectra at room temperature of the three samples of CdTe NCs studied by Morello et al, 2007b. Gray lines are the best fit to the convolution of three Gaussian curves for the first three absorption peaks. Reprinted with permission from Morello, G.; De Giorgi, M.; Kudera, S.; Manna, L.; Cingolani, R. \& Anni, M., Temperature and size dependence of nonradiative relaxation and exciton-phonon coupling in colloidal CdTe quantum dots. J. Phys. Chem. C, 111, 5846-5849. Copyright (2012) American Chemical Society.

Thermal escape is not the only non radiative process featuring colloidal nanocrystals. We have to mention al least two other phenomena contributing to the global lowering of the PL, namely trapping at defect surface states and Auger processes. They are just partially dependent on the temperature and for this reason they are shifty and difficult to analyse.

\subsection{Auger-like interactions}

While the intrinsic decay of singly excited NCs is generally due to the e-h recombination via photon emission, the deactivation of two e-h pairs contemporarily living into the dot is dominated by non radiative Auger recombination. In such a case, the excess energy is not 
released as a photon but is transferred to a third particle (an electron, a hole or an exciton) that is re-excited to higher energy states. The efficiency of Auger processes, which are mediated by Coulomb electron-electron interactions, differs greatly between the atomic and the bulk semiconductor case. In atomic systems (the extreme case of a nanocrystal), for which the electron-electron coupling is much stronger than the electron-photon coupling, the rates of Auger transitions are significantly greater than the rates of the radiative transitions. As a result, the decay of the multi-electron states is dominated by Auger processes. Their efficiency is greatly reduced in bulk materials because of the reduced Coulomb e-e coupling and kinematic restrictions imposed by energy and momentum conservation. As the carriers (electrons and/or holes) occupying higher energy states have larger momentum respect to the ones lying at the band edge, the probability of Auger recombination is near to zero. In order to be allowed, Auger recombination must involve electrons and holes having a high momentum in their lowest energy state, meaning that a rapid spatial variation of the wavefunctions is required. Such situation is absent in bulk semiconductors which are characterized by negligible Auger recombination rates. The situation changes, however, in nanoparticles in which the abrupt truncation of the wavefunctions at the borders of the NCs makes possible high momenta also for the lowest wavefunctions without nodes. The collapse of the restrictions about the momentum conservation makes nanocrystals the ideal candidates for the exploitation of a physical phenomenon sought for a long time, namely the "direct carrier multiplication" (DCM). It consists in the generation of multiple excitons by using the excess energy possessed by a single electron-hole pair excited at higher energy levels (Califano et al., 2004a, 2004b; Velizhanin \& Piryatinski, 2011). Following the concept of Califano et al. (Califano et al., 2004a) such process can be considered an inverse Auger recombination for which a highly excited carrier decaying into its ground state is able to excite a valence electron, thus producing a second e-h pair (see figure 9). In principle, DCM could happen every time the excess energy $\Delta \mathrm{E}$ exceeds the energy gap $\mathrm{E}_{\mathrm{g}}$; in the reality it must compete with phonon scattering, radiative recombination and Auger cooling (this latter process foresees the transfer of the excess energy of an electron to a hole by Coulomb scattering leading to the jump of the hole to deeper valence states as depicted in figure 9) and in general an energetic threshold is associated. In the bulk, due to the restrictions of momentum and energy conservation such threshold could reach very high values (up to $1 \mathrm{eV}$ (Harrison et al., 1999; Wolf et al., 1998)). In nanocrystals, on the contrary, the overcoming of the momentum restrictions makes DCM process possible with threshold energy close to $E_{g}$. In the last four/five years numerous researchers have claimed the reached conditions for carrier multiplication in a number of different semiconductor nanomaterials. Since the first studies the most promising systems, on this regard, seemed to be PbSe (Velizhanin \& Piryatinski, 2011) followed by CdSe (Califano et al., 2004a, 2004b; Lin et al., 2011), InAs (Schaller et al., 2007), InP (Stubbs et al., 2010), Si (Gali et al., 2011), Sn (Allan \& Delerue, 2011). It should be noted, however, that the publication of some works reporting several discrepancies among them about the exact determination of the efficiency of carrier multiplication, indicated that the experimental conditions and managements rather than the actual material type were responsible of some higher and/or lower measured thresholds (Nair et al., 2011; Trinh et al., 2011; McGuire et al., 2010; Rabani \& Baer, 2010). Nowadays, the controversies seem to be far away to be solved although the publication of recent works which attempt to unify the main results. Nevertheless the global significance of the discovery can not be undermined, and the great potential of DCM in technological exploitation well justifies the current exited debate. 


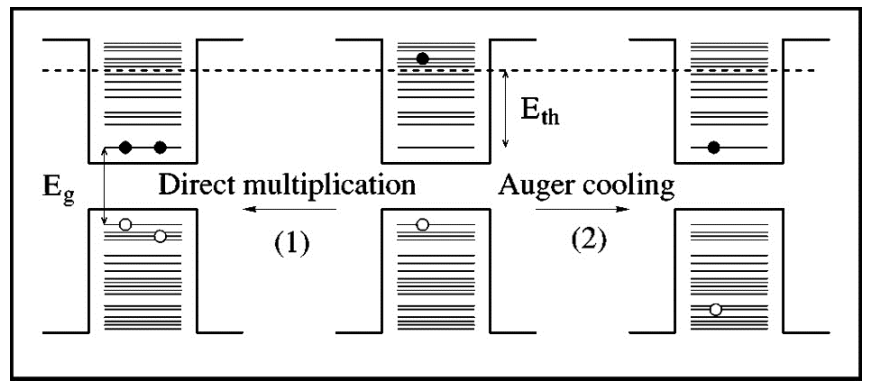

Fig. 9. Schematics of the main hot electron relaxation pathways: Direct Carrier Multiplication (1) and Auger Cooling (2). Reprinted with permission from Califano M.; Zunger A.\& Franceschetti A., Direct carrier multiplication due to inverse Auger scattering in CdSe quantum dots. Appl. Phys. Lett., 84, 2409-2411. Copyright (2012), American Institute of Physics.

\subsection{Surface-related properties}

The surface plays a nontrivial role in defining the optical properties of NCs, especially for what concerns the existence of surface states and their influence on the carrier dynamics. The efficiency of the Auger processes, for instance, may be affected by means of trapping at such states (Cretì et al., 2007). This has important implications on the carrier cooling from higher excited states to the band-edge, which depends on the Auger efficiency, as demonstrated by Klimov and co-workers (Achermann et al., 2006). In their study, for instance, they showed that in nanorods multicarrier generation can cause an Auger heating, consisting in the increase of the energy of an exciton, as a consequence of an energy transfer event deriving from a second exciton recombination. The consequent increase in carrier temperature competes with the more classic phonon emission as a relaxation channel, and decreases the carrier cooling in the high excitation density regime. On the other hand, the surface of NCs constitutes the most probable place to be affected by defect and/or charge accumulation. The reason lies on the fact that in nanostructures the surface to volume ratio is greater with respect to other bulky systems, so that in the strong confinement regime the number of atoms on the surface could reach values larger than the internal ones. Moreover, these atoms present dangling bonds leading to an unavoidable charge imbalance and then to a very high degree of reactivity with the surrounding ambient. As a consequence, the surface atoms constitute the real weak point of each nanoparticle on what concerns the exciton recombination, and a high quality surface reconstruction (passivation) is mandatory in order to obtain good optical performances. The most traditional and easy way to produce a good passivation is to cover the bare surface of nanocrystals with an organic capping layer with the aim to remove potential charge accumulation sites (Bertoni et al., 2007; Cao et al., 2007; Kairdolf et al., 2008; Kalyuzhny \& Murray, 2007; Puzde et al., 2004; Sharma et al., 2009;). A major advance in the improving of optical performances has been reached by surface passivation with an outer solid state material deposited onto the active one and acting as a protective layer (shell). This way, the good choice of the shell could allow for an almost complete reconstruction of the superficial atoms thanks to a good lattice constant matching between the two materials (Isnaeni et al., 2011; Pandey \& Guyot-Sionnest, 2008; Zhang et al., 2010; Zheng et al., 2010). 
It has been shown that the presence of hole surface states can affect the actual order of the single states in the fine structure distribution of the ground state (Califano et al., 2005). Depending on the energetic range covered we can refer to two kinds surface defect states. First, the existence of dangling (unpassivated) bonds on the surface introduces trap states, lying in the forbidden band-gap energy, that can be radiative or not. If they radiate, a typical red shifted (of some hundreds of $\mathrm{meV}$ ) shoulder on the main intrinsic PL spectrum appears (Landes et al., 2001; Underwood et al., 2001; Lim et al., 2008); if they act as non radiative centres, the reduction of the PL quantum yield is the typically observed effect (Burda et al., 2001; Baker \& Kamat, 2010). The growth of a shell usually overcomes the formation of such deep trap states. The second class of traps consists of surface states inducing smaller red shift, typically of the order of a few tens of meV (for this reason they are referred to as shallow trap states), and it may be correlated to the different atomic arrangement of the surface with respect the inside of the dots. All these superficial effects also depend on the size of the nanocrystals, since the fraction of superficial atoms increases as the size decreases and than they define a lower limit attainable for the size of high quality nanocrystals.

In general, surface states are prevalently studied by means of transient absorption measurements (Burda et al., 2001), where the tuning of the probe wavelengths offers the possibility to investigate energies over a very broad range (from UV to NIR on the same sample). If on one hand pump-probe technique allows for discovering numerous properties on what concerns the distribution of trap and intrinsic states, as well as their filling and depopulation dynamics, on the other hand it provides few information about their actual weight in influencing the optical performances of potential devices having nanocrystals as active media. An efficient way to carry out such investigation is to study the dynamics of the radiative relaxation of nanocrystals as a function of the surface passivation. In principle, such a study requires the analysis of the PL decay on a time scale spanning a broad range from picoseconds to microseconds. Great part of the literature regards the analysis of long living radiative emission from deep trap states located at energies well below the lowest optically allowed one. These states present lifetimes on the order of micro-milliseconds depending on their origin and the nature of the host material (Lim et al., 2008). Few works report on the role of shallow surface states on the luminescence properties of NCs and most of them do not discern the nature of the multiple emissive states characteristic, for instance, of nanocrystals having wurtzite structure. As explained in Section 2 the fine structure of CdSe spherical nanocrystals presents a manifold of bright states lying at energies higher than the lowest dark state, complicating the exact determination of the dynamics of the single states. It is obvious that the presence of surface trap states further contributes to complicate the situation. Several theoretical studies report the lifetimes expected for the dark and the bright states, together with the expected effects resulting from mixing of dark and surface states on these lifetimes (Califano et al., 2005). A potentially successful method for discerning the different emitting states (either intrinsic or not) would consists in the optical characterization at single nanoparticle level. However, some intrinsic limitations makes such measurements inadequate. Dynamical intermittent emission and spectral diffusion heavily contribute to cancel information about the actual emission energy and the decay time. 
As for the steady-state optical properties, the most complete lifetime studies on colloidal nanocrystals regard CdSe. In what follows, we recall a treatment on the impact of shallow surface states on the optical properties of CdSe NCs, especially about their radiative relaxation properties, as reported by Morello et al. (Morello et al., 2007a). In general, radiative relaxation channels in CdSe NCs have been investigated by time resolved photoluminescence (TRPL), typically on the nanosecond time scale. These experiments usually exhibit bi-exponential decay traces (Javier et al., 2003; Wang et al., 2003). The shortest lifetime is of the order of several nanoseconds, whereas the longest one is on the time scale of tens of nanoseconds. The origin of these processes (and other longer up to microseconds) is still matter of debate. The longest lifetime is usually attributed to surface states emission (Wang et al., 2003), whereas non-exponential traces, in the same temporal range (Schöps et al., 2006), have been explained in terms of superposition of bright and dark states, and of incomplete surface passivation. Relaxation processes on the microsecond time scale have also been observed and associated to dark state emission (Crooker et al., 2003). Califano et al. (Califano et al., 2005) have shown that the microsecond decay time is actually due to dark-bright state emission induced by the presence of surface states, while the dark state has been predicted to have a millisecond lifetime. At the single dot level, TRPL on CdSe QDs has revealed nanosecond lifetimes (Labeau et al., 2003) (probably, emission from dark-bright states) as well as nonexponential decays arising from fluctuating non radiative relaxation channels (Fisher et al., 2004; Schlegel et al., 2002).

An interesting issue concerns the role of the bright states on the temporal dynamics of the PL, and their interplay with dark and/or surface states. These properties have been investigated for the first time by Wang et al. (Wang et al., 2006) who showed the carrier relaxation from bright to dark and surface defect states. Since such kind of relaxation is predicted to be faster than the natural radiative emission lifetime (less than $100 \mathrm{ps}$ ) the role of $\pm 1 \mathrm{U}$ and $\pm 1^{\mathrm{L}}$ bright intrinsic states (see Section 2 and Efros et al., 1996 for details) in presence of emitting surface states has been only postulated (Bawendi et al., 1992; Jungnickel \& Henneberger, 1996). As representative examples we can cite Bawendi et al. (Bawendi et al., 1992) who have showed transient emission from CdSe QDs involving surface states. These authors distinguished both a short lifetime of the order of their time resolution (about $100 \mathrm{ps}$ ) probably arising from intrinsic emission, and a temperaturedependent interplay between the band-edge and surface states. Jungnickel and Henneberger (Jungnickel \& Henneberger, 1996) have found similar transient behaviour on the same time scale, stressing the long decay time of radiative surface state emission. de Mello Donegá et al. (de Mello Donegà et al., 2006) have investigated the temperature dependence of the exciton lifetime in CdSe QDs, finding evidence for a fast component in the time trace in the low temperature "radiative regime", again within the temporal resolution of their system (700 ps). Such contribution was ascribed to rapid carrier thermalization from bright to dark states. A reliable study able to resolve fast emission from bright states (relaxing into the surface ones) can be performed by means of a streak camera (temporal resolution below $10 \mathrm{ps}$ ), with the possibility to find evidence for the thermal evolution of the population of the single states (Morello et al. 2007a). As a case study, we report here a comparative investigation between core CdSe and core/shell CdSe/ZnS NCs having the same core dimension by TRPL measurements in the temperature range of 15-300 K (Morello et al., 2007a).

In figure 10 (left panel) the temporal evolution of CdSe/ZnS QDs PL spectra at $20 \mathrm{~K}$ is shown. After $1.7 \mathrm{~ns}$ a small red shift is observed, completed after $12 \mathrm{~ns}$. By analysing the 
spectrum at 0 ps delay (figure 11) it is possible to discern the single emitting entities responsible for the observed PL. In this particular case the PL results as a convolution of 3 emitting states lying at different energies, as shown in figure 11. The dynamical red shift, therefore, reveals the different dynamics the 3 states undergo. In the study of Morello et al. (Morello et al., 2007a) two samples of NCs were investigated, namely CdSe and CdSe/ZnS (both samples having the same core dimensions). They found the following energetic separations among the states: $E_{1,2}=21 \mathrm{meV}$ and $E_{2,3}=16 \mathrm{meV}$ for core NCs; $E_{1,2}=21 \mathrm{meV}$ and $\mathrm{E}_{2,3}=13 \mathrm{meV}$ for core/shell NCs.
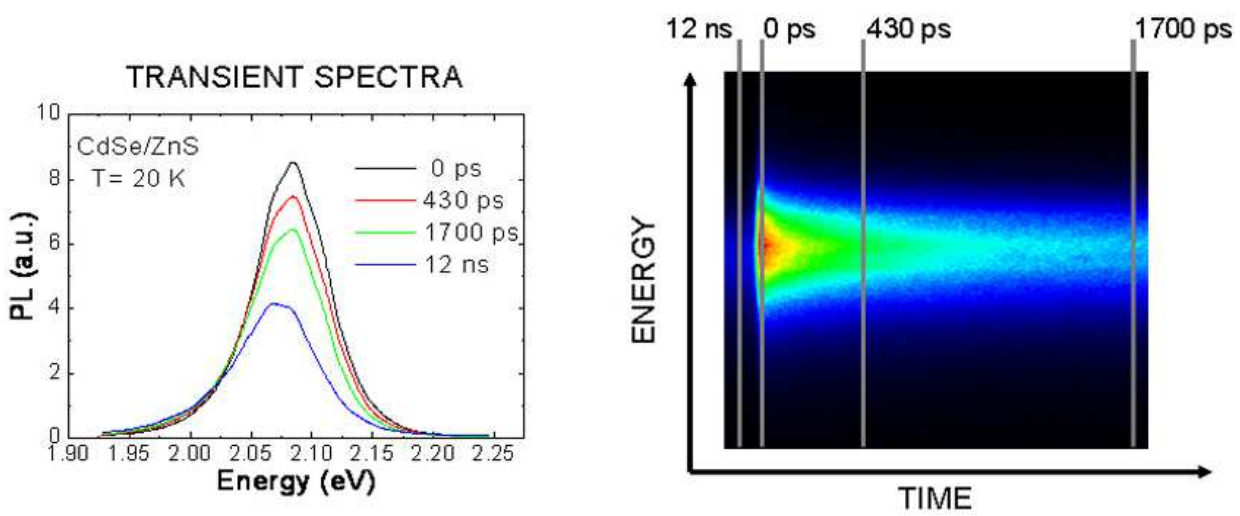

Fig. 10. Left panel: transient PL spectra of CdSe/ZnS NCs at $20 \mathrm{~K}$ from 0 ps to 12 ns after the pump pulse. Right panel: PL decay trace showing the temporal slices reported in the left panel.

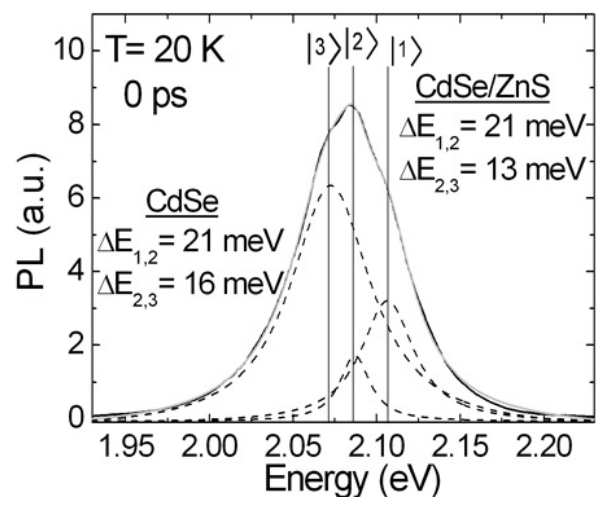

Fig. 11. PL spectrum at $20 \mathrm{~K}$ of CdSe/ZnS NCs taken at 0 ps delay looked as the result of three superposed transitions. 


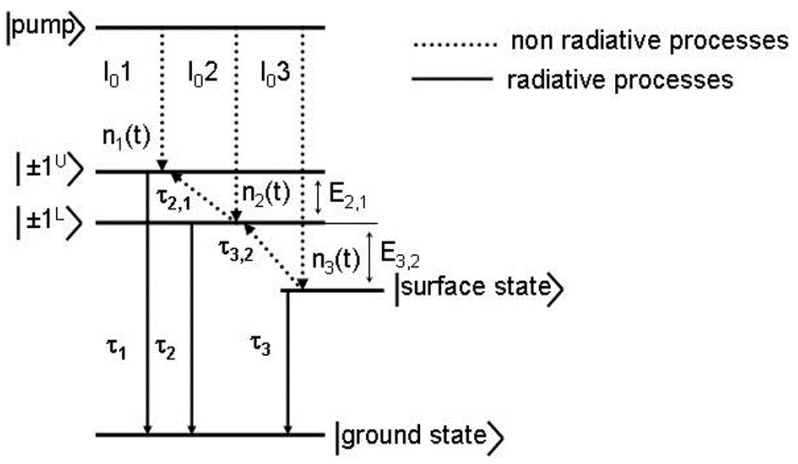

Fig. 12. Schematics of the radiative and non radiative processes occurring among two intrinsic and a surface state in CdSe nanocrystals in the low temperature range (10-70 K). Reprinted with permission from Morello, G.; Anni, M.; Cozzoli, P. D.; Manna, L.; Cingolani, R. \& De Giorgi, M., Picosecond Photoluminescence Decay Time in Colloidal Nanocrystals: The Role of Intrinsic and Surface States. J. Phys. Chem. C , 111, 10541- 10545. Copyright (2012) American Chemical Society.

The PL time decay for core and core/shell samples (figure 10, right panel, shows an image of the time trace as recorded by the Streak Camera) was well reproduced by a triexponential decay function for both the samples studied in a temperature range of 15-300 K:

$$
I(t)=A_{1} \cdot e^{-\left(t-t_{0}\right) / t_{1}}+A_{2} \cdot e^{-\left(t-t_{0}\right) / t_{2}}+A_{3} \cdot e^{-\left(t-t_{0}\right) / t_{3}}
$$

where $t_{0}$ is the delay at which $I(t)$ is maximum, $t_{1}, t_{2}, t_{3}$ are the lifetimes and $A_{1}, A_{2}, A_{3}$ are the weights of each process, respectively. The time constants $t_{1}$ and $t_{2}$ were the typical carrier relaxation times from intrinsic bright states of the fine structure of spherical CdSe NCs into the surface defect states (Wang et al., 2006), and $t_{3}$ was comparable with typical lifetime of surface-related emission in CdSe NCs (Wang et al., 2006). Moreover, the extracted energy splitting $E_{1,2}$ was the same in core and core/shell sample, similar to the splitting between the lowest bright states $\pm 1 \mathrm{U}$ and $\pm 1 \mathrm{~L}$ in CdSe QDs $\left(20 \mathrm{meV}\right.$ ) (Efros et al., 1996), whereas $\mathrm{E}_{2,3}$ was different in the two studied samples, revealing an extrinsic nature of the reddest transition. A credible origin was ascribed to surface states, also considering that the dark state lifetime should be of the order of micro-milliseconds. From temperature dependent measurements it was possible to analyse the contribution of the single emitting states by integration of the single exponential terms of eq. 39 :

$$
\begin{aligned}
& I_{1}(T)=\int_{0}^{\infty} A_{1} \cdot e^{-\left(t-t_{0}\right) / t_{1}} d t, \\
& I_{2}(T)=\int_{0}^{\infty} A_{2} \cdot e^{-\left(t-t_{0}\right) / t_{2}} d t, \\
& I_{3}(T)=\int_{0}^{\infty} A_{3} \cdot e^{-\left(t-t_{0}\right) / t_{3}} d t .
\end{aligned}
$$




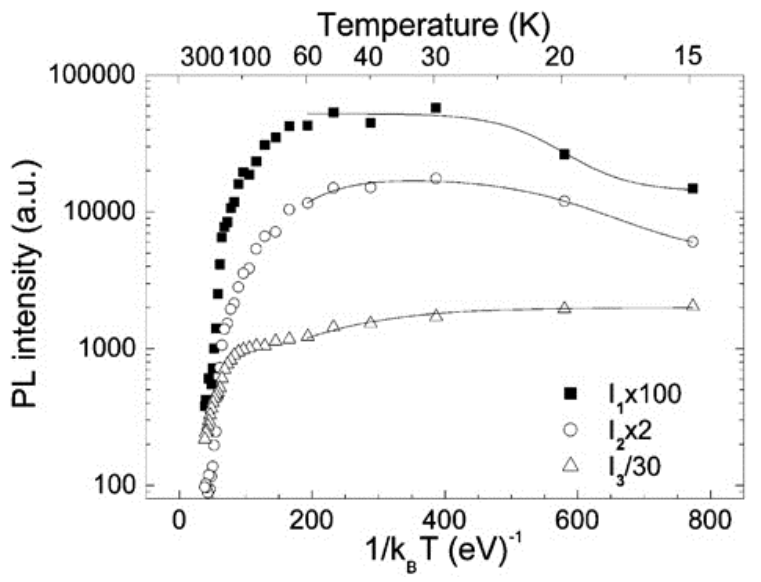

Fig. 13. PL intensity of the three states as a function of temperature for CdSe/ZnS QDs. The continuous lines are the fit curves to eqs 42. Reprinted with permission from Morello, G.; Anni, M.; Cozzoli, P. D.; Manna, L.; Cingolani, R. \& De Giorgi, M., Picosecond Photoluminescence Decay Time in Colloidal Nanocrystals: The Role of Intrinsic and Surface States. J. Phys. Chem. C , 111, 10541- 10545. Copyright (2012) American Chemical Society.

Figure 13 shows the temperature dependence of the PL intensities $I_{1}, I_{2}$ and $I_{3}$ for the three states from which it is clear the existence of several thermal regimes of population and depopulation processes. The PL thermal increase for $I_{1}$ and $I_{2}$ in the range of $15-70 \mathrm{~K}$ suggests that thermally induced population of the high energy states occurs, fed by the lowest energy state. At higher temperatures, on the other hand, the overall PL intensity strongly decreases, indicating the occurrence of thermal activation of non radiative relaxation channels, such as thermal escape induced by multiple LO phonons absorption. The dynamics occurring at low temperature (up to $70 \mathrm{~K}$ ) was well explained by considering a four-level system, as depicted in figure 12. After laser excitation, carriers relax non radiatively into states $\pm 1 \mathrm{U}, \pm 1 \mathrm{~L}$ and into surface states, from which they relax radiatively. The lifetime of states $\pm 1 \mathrm{U}$ and $\pm 1 \mathrm{~L}$ is shorter than the intrinsic radiative decay time, because of the fast carrier relaxation into the surface state, which instead can emits with its intrinsic radiative decay time. According to this picture, the two shortest lifetimes were attributed to carriers relaxing from $\pm 1 \mathrm{U}$ and $\pm 1 \mathrm{~L}$ bright states into the surface one. The effectiveness of the model could be verified by solving the equation system associated to thermal population and depopulation of the states with non radiative lifetime:

$$
\left\{\begin{array}{l}
\frac{d n_{3}(t)}{d t}=n_{03} \delta(t)-\frac{n_{3}(t)}{\tau_{3,2}} e^{-E_{3,2} / k_{B} T}-\frac{n_{3}(t)}{\tau_{3}} \\
\frac{d n_{2}(t)}{d t}=n_{02} \delta(t)-\frac{n_{2}(t)}{\tau_{2,1}} e^{-E_{2,1} / k_{B} T}+\frac{n_{3}(t)}{\tau_{3,2}} e^{-E_{3,2} / k_{B} T}-\frac{n_{2}(t)}{\tau_{2}} \\
\frac{d n_{1}(t)}{d t}=n_{01} \delta(t)+\frac{n_{2}(t)}{\tau_{2,1}} e^{-E_{2,1} / k_{B} T}-\frac{n_{1}(t)}{\tau_{1}}
\end{array}\right.
$$


where $\mathrm{n}_{01}, \mathrm{n}_{02}$, and $\mathrm{n}_{03}$ represent the respective initial population of the three states as generated by the laser pulse (the latter is treated as a $\delta$ function because the pump pulse is much shorter than all the processes considered here); $\tau_{1}, \tau_{2}$, and $\tau_{3}$ are the intrinsic radiative decay times of the states; $1 / \tau_{2,1}$ and $1 / \tau_{3,2}$ are the depletion rates of the state $\pm 1^{\mathrm{L}}$ and the surface state, respectively; and $\mathrm{E}_{2,1}$ and $\mathrm{E}_{3,2}$ are the energy separations among the three states as indicated in figure 12. By solving the system, we obtain the expressions for the timedependent populations $\mathrm{n}_{1}(\mathrm{t}), \mathrm{n}_{2}(\mathrm{t})$, and $\mathrm{n}_{3}(\mathrm{t})$ of the states involved in the emission process. Thus, the PL integrated areas $\mathrm{I}_{1}(\mathrm{~T}), \mathrm{I}_{2}(\mathrm{~T})$, and $\mathrm{I}_{3}(\mathrm{~T})$ are as follows:

$$
\begin{aligned}
& I_{1}(T)=\int_{0}^{\infty} \frac{n_{1}(t)}{\tau_{1}} d t=I_{01}+\frac{\tau_{2}}{\tau_{2,1}} e^{-E_{2,1} / k_{B} T}\left(I_{02}+I_{03} \rho\right), \\
& I_{2}(T)=\int_{0}^{\infty} \frac{n_{2}(t)}{\tau_{2}} d t=\frac{I_{02}+I_{03} \rho}{1+\frac{\tau_{2}}{\tau_{2,1}} e^{-E_{2,1} / k_{B} T}}, \\
& I_{3}(T)=\int_{0}^{\infty} \frac{n_{3}(t)}{\tau_{3}} d t=\frac{I_{03}}{1+\frac{\tau_{3}}{\tau_{3,2}} e^{-E_{3,2} / k_{B} T}},
\end{aligned}
$$

where

$$
\rho=\frac{\frac{\tau_{3}}{\tau_{3,2}} e^{-E_{3,2} / k_{B} T}}{1+\frac{\tau_{3}}{\tau_{3,2}} e^{-E_{3,2} / k_{B} T}} .
$$

The three expressions represent the theoretical counterpart of eqs. 40 and could be, thus, employed to fit the experimental data of figure 13 in the low temperature range with the aim to extract the actual energetic separation among the states and the relative lifetimes.

\section{Conclusion}

The importance of spherical nanocrystals relies of the fact that they represent the perfect model of a quantum confined system. This leads to consider these nanoparticles ideal for the study of the fundamental physics at the basis of the quantum mechanics and several applications in technological field. In the latter context, chemically synthesized nanoparticles are the most promising candidates to be used as active media in optoelectronic devices, due to the relatively easy methods of production and to the versatility of the colloidal solution form in which they appear. As pioneering nano-objects, spherical nanocrystals have attracted remarkable attention by researchers and constitute the most studied systems in terms of opto-electric properties. In this chapter we covered several aspects on what concerns their optical properties. We started with an overview of the main consequences resulting from the progressive shrinking of the spatial extension of a solid system, and of the implications on the modulation of the DOS function and the quantization of the energy. Particular attention has been dedicated to the textbook case of the Schrödinger equation in one dimension. A brief excursus has been dedicated to the electronic 
configuration of the fine structure of the ground state in wurtzite and cubic crystal structure. As examples of interest we have tackled the cases of CdSe (wurtzite) and CdTe (cubic) nanocrystals since they are, in absolute, the most studied systems, following the pioneering works of Efros. Regarding the optical properties, we have concentrated our attention to the effects deteriorating the optical performances in terms of quantum efficiency (especially acting at room temperature), which depend on both intrinsic and extrinsic factors. The firsts include all the effects which can not be avoided by any synthesis improvement, because they are typical of the physics of the material, such as exciton-phonon coupling and Auger effects. The seconds are related to the global purity of the nanocrystals in terms of crystalline structure and surface quality. The latter, in particular, constitutes the major contributor to the observation of poor emission performances or, sometimes, spurious effects. A review of the main studies about this topic has been provided with a particular attention to the methods for studying and discerning the role of surface states. The reported examples wanted to stress the importance of a critical point in the definition of the optical performances of colloidal nanocrystals which is the presence of surface states. Their study is constantly evolving as well as the attempts to reduce their negative effects by improving the chemical methods. The first is preparatory for the second and often the second contributes to complicate the first, such that nowadays the duel seems to be far away to end.

\section{Acknowledgments}

The author acknowledges M. De Giorgi, L. Manna, M. Anni, R. Cingolani, P. D. Cozzoli, for fundamental contributions and P. Cazzato for precious technical assistance. Finally, he wants to thank the CBN centre of IIT for the complete funding of this work

\section{References}

Achermann, M.; Bartko, A. P.; Hollingsworth, J. A. \& Klimov, V. I., (2006). The effect of Auger heating on intraband carrier relaxation in semiconductor quantum rods. Nat. Phys., Vol. 2, No. 8, pp. 557-561.

Allan, G. \& Delerue, C., (2011). Optimization of Carrier Multiplication for More Effcient Solar Cells: The Case of Sn Quantum Dots. ACS Nano, Vol. 5, No. 9, pp. 7318-7323.

Anikeeva, P. O.; Halpert, J. E.; Bawendi, M. G. \& Bulović, V. (2009). Quantum Dot LightEmitting Devices with Electroluminescence Tunable over the Entire Visible Spectrum. Nano Letters, Vol. 9, No. 7, (September 2009), pp. 2532-2536.

Baker, D. R. \& Kamat, P. V., (2010). Tuning the Emission of CdSe Quantum Dots by Controlled Trap Enhancement. Langmuir, Vol. 26, No. 13, pp. 11272-11276.

Bawendi, M. G.; Carroll, P. J.; Wilson, W. L.\& Brus, L. E., (1992). Luminescence properties of CdSe quantum crystallites: Resonance between interior and surface localized states. J. Chem. Phys., Vol. 96, No. 2, pp. 946-954.

Bertoni, C.; Gallardo, D.; Dunn, S.; Gaponik, N. \& Eychmüller, A., (2007). Fabrication and characterization of red-emitting electroluminescent devices based on thiolstabilized semiconductor nanocrystals. Appl. Phys. Lett., Vol. 90, 034107-034109.

Burda, C.; Link, S.; Mohamed, M. \& El-Sayed, M., (2001). The relaxation pathways of CdSe nanoparticles monitored with femtosecond time-resolution from the visible to the 
IR: Assignment of the transient features by carrier quenching. J. Phys. Chem. B, Vol. 105, No. 49. pp. 12286-12292.

Califano M; Zunger A \& Franceschetti A., (2004a). Direct carrier multiplication due to inverse Auger scattering in CdSe quantum dots. Appl. Phys. Lett., Vol. 84, No. 13, pp. 2409-2411.

Califano M; Zunger A \& Franceschetti A., (2004b). Efficient inverse Auger recombination at threshold in CdSe nanocrystals. Nano Lett., Vol. 4, No. 3, pp. 525-531.

Califano, M.; Franceschetti, A. \& Zunger, A., (2005). Temperature Dependence of Excitonic Radiative Decay in CdSe Quantum Dots: The Role of Surface Hole Traps. Nano Lett., Vol. 5, No. 12, pp. 2360-2364.

Cao, X.; Li, C. M.; Bao, H.; Bao, Q. \& Dong, H., (2007). Fabrication of Strongly Fluorescent Quantum Dot-Polymer Composite in Aqueous Solution. Chem. Mater., Vol. 19, pp. 3773-3779.

Caruge, J. M.; Halpert, J. E.; Wood, V.; Bulović, V. \& Bawendi, M. G. (2008). Colloidal quantum-dot light-emitting diodes with metal-oxide charge transport layers. Nature Photonics, Vol. 2, pp. 247-250.

Chan, Y.; Caruge, J. M.; Snee, P. T. \& Bawendi, M. G. (2004). Multiexcitonic two-state lasing in a CdSe nanocrystal laser. Applied Physics Letters, Vol. 85, No. 13, (December 2005), pp. 2460-2462.

Cretì, A.; Anni, M.; Zavelani-Rossi, M.; Lanzani, G.; Leo, G.; Della Sala, F.; Manna, L. \& Lomascolo, M., (2005). Ultrafast carrier dynamics in core and core/shell CdSe quantum rods: Role of the surface and interface defects. Phys. Rev. B, Vol. 72, No. 12, pp. 125346-125355.

Creti, A.; Anni, M.; Zavelani-Rossi, M.; Lanzani, G.; Manna, L. \& Lomascolo, M., (2007). Role of defect states on Auger processes in resonantly pumped CdSe nanorods. Appl. Phys. Lett., Vol. 91, No. 9, pp. 093106-093108.

Crooker, S. A.; Barrick, T.; Hollingsworth, J. A. \& Klimov, V. I., (2003). Multiple temperature regimes of radiative decay in CdSe nanocrystal quantum dots: Intrinsic limits to the dark-exciton lifetime. Appl. Phys. Lett., Vol. 82, No. 17, pp. 2793-2795.

de Broglie, L. Recherches sur la théorie des quanta (Researches on the quantum theory), Thesis, Paris.

de Broglie, L. (1925), Ann. Phys. (Paris), Vol. 3, pp. 22.

de Mello Donega, C.; Bode, M. \& Meijerink, A., (2006). Size- and temperature-dependence of exciton lifetimes in CdSe quantum dots. Phys. Rev. B, Vol. 74, No. 8, pp. 085320085328.

Davies, J. H. (1998).Physics of Low Dimensional Semiconductors, Cambridge University Press, ISBN 0-521-48148-1, Cambridge.

Deka, S.; Quarta, A.; Lupo, M. G.; Falqui, A.; Boninelli, S.; Giannini, C.; Morello, G.; De Giorgi, M.; Lanzani, G.; Spinella, C.; Cingolani, R.; Pellegrino, T. \& Manna, L. (2009). CdSe/CdS/ZnS Double Shell Nanorods with High Photoluminescence Efficiency and Their Exploitation As Biolabeling Probes. J. Am. Che. Soc., Vol. 131, pp. 2948-2958. 
Efros, Al. L.; Rosen, M.; Kuno, M.; Nirmal, M.; Norris, D. J.\& Bawendi, M. (1996) Band-edge exciton in quantum dots of semiconductors with a degenerate valence band: Dark and bright exciton states. Phys. Rev. B, Vol. 54, No. 7, pp. 4843-4856.

Efros, Al. L. \& Rosen, M. (2000). The electronics structure of semiconductor nanocrystals. Annu. Rev. Mater. Sci., Vol. 30, pp. 475-521.

Empedocles, S. A. \& Bawendi, M. G. (1999). Influence of Spectral Diffusion on the Line Shapes of Single CdSe Nanocrystallite Quantum Dots. J. Phys. Chem. B, Vol. 103, No. 11, pp. 1826-1830.

Fiore, A.; Mastria, R.; Lupo, M. G.; Lanzani, G.; Giannini, C.; Carlino, E.; Morello, G.; De Giorgi, M.; Li, Y.; Cingolani, R. \& Manna, L. (2009). Tetrapod-Shaped Colloidal Nanocrystals of II-VI Semiconductors Prepared by Seeded Growth. J. Am. Chem. Soc., Vol. 131, No. 6, pp. 2274-2282.

Fisher, B. R.; Eisler, H. J.; Stott, N. E. \& Bawendi, M. G., (2004). Emission intensity dependence and single-exponential behavior in single colloidal quantum dot fluorescence lifetimes. J. Phys. Chem. B, Vol. 108, No. 1, pp. 143-148.

Gali, A.; Kaxiras, E.; Zimanyi, G. T. \& Meng, S., (2011). Effect of symmetry breaking on the optical absorption of semiconductor nanoparticles. Phys. Rev. B, Vol. 84, 035325035330.

Gaponenko, S. V., (1998). Optical Properties of Semiconductor Nanocrystals (2005). Cambridge University Press, 9780521019231, Cambridge, U.K..

Gindele, F.; Hild, K.; Langbein, W. \& Woggon, U., (2000). Temperature-dependent line widths of single excitons and biexcitons. J. Lumin., Vol. 87-89, pp. 381-383.

Gur, I.; Fromer, N. A.; Geier, M. L. \& Alivisatos, A. P. (2005). Air-Stable All-Inorganic Nanocrystal Solar Cells Processed from Solution. Science, Vol. 310, No. 5747, pp. 462-465.

Guyot-Sionnest, P.; Shim, M.; Matranga, C. \& Hines, M., (1999). Intraband relaxation in CdSe quantum dots. Phys. Rev. B, Vol. 60, No. 4, pp. R2181-R2184.

Harrison, D.; Abram, R. A. \& Brand, S., (1999). Characteristics of impact ionization rates in direct and indirect gap semiconductors. J. Appl. Phys., Vol. 85, No. 12, pp. 81868192.

Hewa-Kasakarage, N. N.; El-Khoury, P. Z.; Tarnovsky, A. N.; Kirsanova, M.; Nemitz, I.; Nemchinov, A. \& Zamkov, M. (2010). Ultrafast carrier dynamics in type II ZnSe/CdS/ZnSe nanobarbells. ACS Nano, Vol. 4, pp. 1837-1844.

Huynh, W. U.; Dittmer, J.J. \& Alivisatos, A. P. (2002). Hybrid Nanorod-Polymer Solar Cells. Science, Vol. 295 (October 2011), pp. 2425-2427.

Isnaeni; Kim, K. H.; Nguyen, D. L.; Lim, H.; Pham, T. N. \& Cho, Y.-H., (2011). Shell layer dependence of photoblinking in CdSe/ZnSe/ZnS quantum dots. Appl. Phys. Lett., Vol. 98, No. 1, pp. 12109-12111.

Javier, A.; Magana, D.; Jennings, T. \& Strouse, G. F., (2003). Nanosecond exciton recombination dynamics in colloidal CdSe quantum dots under ambient conditions. Appl. Phys. Lett., Vol. 83, No. 7, pp. 1423-1425.

Jungnickel, V. \& Henneberger, F., (1996). Luminescence related processes in semiconductor nanocrystals - The strong confinement regime. J. Lumin., Vol. 70, pp. 238-252. 
Kairdolf, B. A.; Smith, A. M. \& Nie, S, (2007). One-Pot Synthesis, Encapsulation, and Solubilization of Size-Tuned Quantum Dots with Amphiphilic Multidentate Ligands. J. Am. Chem. Soc., Vol. 130, pp. 12866-12867.

Kalyuzhny, G. \& Murray, R. W., (2005). Ligand Effects on Optical Properties of CdSe Nanocrystals. J. Phys. Chem. B, Vol. 109, pp. 7012-7021.

Kim, J.; Wong, C. Y. \& Scholes, G. D. (2009). Exciton fine structure and spin relaxation in semiconductor colloidal quantum dots. Acc. Chem. Res., Vol. 42, No. 8, pp. 10371046.

Kim, S.; Fisher, B.; Eisler, H.-J. \& Bawendi, M. G. (2003). Type-II Quantum Dots: $\mathrm{CdTe} / \mathrm{CdSe}$ (Core/Shell) and CdSe/ZnTe(Core/Shell) Heterostructures. Journal of the American Chemical Society, Vol. 125, (September 2003), pp. 11466-11467.

Klimov, V. I. \& McBranch, D. W., (1998). Femtosecond 1P-to-1S electron relaxation in strongly confined semiconductor nanocrystals. Phys. Rev. Lett., Vol. 80, No. 18, pp. 4028-4031.

Klimov, V. I., (2000). Optical nonlinearities and ultrafast carrier dynamics in semiconductor nanocrystals. J. Phys. Chem. B, Vol. 104, No. 26, pp. 6112-6123.

Klimov, V. I.; Mikhailovsky, A. A.; Xu, S.; Malko, A.; Hollingsworth, J. A.; Leatherdale, C. A.; Eisler,H.-J. \& Bawendi, M. G. (2000). Optical Gain and Stimulated Emission in Nanocrystal Quantum Dots. Science, Vol. 290, (October 2000), pp. 314-317.

Klimov, V. I.; Ivanov, S. A.; Nanda, J.; Achermann, M.; Bezel, I.; McGuire, J. A. \& Piryatinski, A. (2007). Single-exciton optical gain in semiconductor nanocrystals. Nature, Vol. 447, (May 2007), pp. 441-446.

Kong, L. M.; Cai, J. F.; Wu, Z. W.; Gong, Z.; Niu, Z. C. \& Feng, Z. C. (2006). Time-resolved photoluminescence spectra of self-assembled InAs/GaAs quantum dots. Thin Solid Films, Vol. 498, No. 1-2, pp. 188-192.

Krahne, R.; Morello, G.; Figuerola, A.; George, C.; Deka, S. \& Manna, L. (2011). Physical properties of elongated inorganic nanoparticles. Phys. Rep., Vol. 501, No. 3-5, pp. 75-221.

Labeau, O.; Tamarat, P. \& Lounis, B., (2003). Temperature dependence of the luminescence lifetime of single CdSe/ZnS quantum dots. Phys. Rev. Lett., Vol. 90, No. 25, pp. 257404-257408.

Landes, C. F.; Braun, M. \& El-Sayed, M. A., (2001). On the nanoparticle to molecular size transition: Fluorescence quenching studies. J. Phys. Chem. B, Vol. 105, No. 43, pp. 10554-10558.

Lee, W. Z.; Shu, G. W.; Wang, J. S.; Shen, J. L.; Lin, C. A.; Chang, W. H.; Ruaan, R. C.; Chou, W. C.; Lu, C. H. \& Lee, Y. C., (2005). Recombination dynamics of luminescence in colloidal CdSe/ZrS quantum dots. Nanotechnology, Vol. 16, No. 9, pp. 1517-1521.

Liebler, J. G.; Schmitt-Rink, S. \& Haug, H., (1985). Theory of the absorption tail of wannier excitons in polar semiconductors. J. Lumin., Vol. 34, No. 1-2, pp. 1-7.

Lim, S. J.; Chon, B.; Joo, T. \& Shin, S. K., (2008). Synthesis and Characterization of ZincBlende CdSe-Based Core/Shell Nanocrystals and Their Luminescence in Water. J. Phys. Chem C, Vol. 112, pp. 1744-1747. 
Lin, Z.; Franceschetti, A. \& Lusk, M. T., (2011). Size Dependence of the Multiple Exciton Generation Rate in CdSe Quantum Dots. ACS Nano, Vol. 5, No. 4, pp. 2503-2511.

McGuire, J. A.; Sykora, M.; Joo, J.; Pietryga, J. M. \& Klimov, V. I., (2010). Apparent Versus True Carrier Multiplication Yields in Semiconductor Nanocrystals. Nano Lett., Vol. 10, pp. 2049-2057.

Michalet, X. ; Pinaud, F. ; Lacoste, T. D.; Dahan, M.; Bruchez, M. P. ; Alivisatos, A. P. \& Weiss, S. (2001). Properties of Fluorescent Semiconductor Nanocrystals and their Application to Biological Labeling. Single Mol., Vol. 2, pp. 261-276.

Millo, O.; Katz, D.; Steiner, D.; Rothenberg, E.; Mokari, T.; Kazes, M. \& Banin, U. (2004). Charging and quantum size effects in tunnelling and optical spectroscopy of CdSe nanorods. Nanotechnology, Vol. 15, pp. R1-R6.

Miszta, K.; Dorfs, D.; Genovese, A.; Kim, M. R. \& Manna, L. (2011). Cation Exchange Reactions in Colloidal Branched Nanocrystals. ACS Nano, Vol. 5, No. 9, pp. 71767183.

Moreels, I.; Rainò, G.; Gomes, R.; Hens, Z.; Stöferle, T. \& Mahrt, R. F., (2011). Band-Edge Exciton Fine Structure of Small, Nearly Spherical Colloidal CdSe/ZnS Quantum Dots. ACS Nano, Vol. 5, No. 10, pp. 8033-8039.

Morello, G.; Anni, M.; Cozzoli, P. D.; Manna, L.; Cingolani, R. \& De Giorgi, M. (2007a). Picosecond Photoluminescence Decay Time in Colloidal Nanocrystals: The Role of Intrinsic and Surface States. J. Phys. Chem. C, Vol. 111, No. 28, pp. 1054110545.

Morello, G.; De Giorgi, M.; Kudera, S.; Manna, L.; Cingolani, R. \& Anni, M., (2007b). Temperature and size dependence of nonradiative relaxation and exciton-phonon coupling in colloidal CdTe quantum dots. J. Phys. Chem. C, Vol. 111, No. 16, pp. 5846-5849.

Muljarov, E. A. \& Zimmermann, R., (2007). Exciton Dephasing in Quantum Dots due to LOPhonon Coupling: An Exactly Solvable Model. Phys. Rev. Lett., Vol. 98, No. 18, pp. 187401-187404.

Nair, G.; Chang, L.-Y.; Geyer, S. M. \& Bawendi, M. G., (2011). Perspective on the Prospects of a Carrier Multiplication Nanocrystal Solar Cell. Nano Lett., Vol. 11, pp. 21452151.

Nomura, S. \& Kobayashi, T., (1992). Exciton-LO-phonon couplings in spherical semiconductor microcrystallites. Phys. Rev. B, Vol. 45, No. 3, pp. 1305-1316.

Norris, D. J.; Efros, Al. L.; Rosen, M. \& Bawendi, M. G. (1996). Size dependence of exciton fine structure in CdSe quantum dots. Phys. Rev. B, Vol. 53, No. 24, pp. 1634716354.

Nozik, A. J., (2001). Spectroscopy and hot electron relaxation dynamics in semiconductor quantum wells and quantum dots. Annu. Rev. Phys. Chem., Vol. 52, pp.193-231.

Oertel, D. C.; Bawendi, M. G.; Arango, A. C. \& Bulović, V. (2005). Photodetectors based on treated CdSe quantum-dot films. Appl. Phys. Lett., Vol. 87, pp. 213505-213507.

Ohmura, H. \& Nakamura, A. (1999). Quantum beats of confined exciton-LO phonon complexes in $\mathrm{CuCl}$ nanocrystals. Phys. Rev. B, Vol. 59, No, 19, pp. 12216-12219. 
Pandey, A. \& Guyot-Sionnest, P., (2008). Slow Electron Cooling in Colloidal Quantum Dots. Science, Vol. 322, No. 5903, pp. 929-932.

Peng, X. G.; Manna, L.; Yang, W. D.; Wickham, J.; Scher, E.; Kadavanich, A. \& Alivisatos, A. P. (2000). Shape control of CdSe nanocrystals. Nature, Vol. 404, No. 6773, pp. 59-61.

Pines, D. (1963), Elementary excitations in solids, W. A. Benjamin Inc., New York.

Puzder, A.; Williamson, A. J.; Zaitseva, N. \& Galli, G., (2004). The Effect of Organic Ligand Binding on the Growth of CdSe Nanoparticles Probed by Ab Initio Calculations. Nano Lett., Vol. 4, No. 12, pp. 2361-2365.

Rabani, E. \& Baer, R., (2010). Theory of multiexciton generation in semiconductor nanocrystals. Chem. Phys. Lett., Vol. 496, pp. 227-235.

Rothenberg, E.; Kazes, M.; Shaviv, E. \& Banin, U., (2005). Electric field induced switching of the fluorescence of single semiconductor quantum rods. Nano Lett., Vol. 5, No. 8, pp. 1581-1586.

Rudin, S.; Reinecke, T. L. \& Segall, B., (1990). Temperature-dependent exciton linewidths in semiconductors. Phys. Rev. B, Vol. 42, No. 17, pp. 11218-11231.

Schaller, R. D.; Pietryga, J. M. \& Klimov, V. I., (2007). Carrier Multiplication in InAs Nanocrystal Quantum Dots with an Onset Defined by the Energy Conservation Limit. Nano Lett., Vol. 7, No. 11, pp. 3469-3476.

Sharma, H.; Sharma, S. N.; Kumar, U.; Singh, V. N.; Mehta, B. R.; Singh, G.; Shivaprasad, S. M. \& Kakkar, R., (2009). Formation of water-soluble and biocompatible TOPOcapped CdSe quantum dots with efficient photoluminescence. J. Mater. Sci.: Mater. Med., Vol. 20, pp. S123-S130.

Schlegel, G.; Bohnenberger, J.; Potapova, I. \& Mews, A. (2002). Fluorescence Decay Time of Single Semiconductor Nanocrystals. Phys. Rev. Lett., Vol. 88, pp. 137401-197404.

Schmitt-Rink, S.; Miller, D.A.B. \& Chemla, D.S., (1987). Theory of the linear and nonlinear optical properties of semiconductor microcrystallites. Phys. Rev. B, Vol. 35, No. 15, pp. 8113-8125.

Schöps, O.; Le Thomas, N.; Woggon, U. \& Artemyev, M. V., (2006). Recombination dynamics of CdTe/CdS core-shell nanocrystals. J. Phys. Chem. B, Vol. 110, No. 5, pp. 2074-2079.

Stubbs, S. K.; Hardman, S. J. O.; Graham, D. M.; Spencer, B. F,; Flavell, W. R.; Glarvey, P.; Masala, O.; Pickett, N. L. \& Binks, D. J., (2010). Efficient carrier multiplication in InP nanoparticles. Phys. Rev. B, Vol. 81, pp. 081303-081306 (R).

Takagahara, T., (1996). Electron-phonon interactions in semiconductor nanocrystals. $J$. Lumin., Vol. 70, No. 1-6, pp. 129-143.

Trallero-Giner, C.; Debernardi, A.; Cardona, M.; Menendez-Proupin, E. \& Ekimov, A. I., (1998). Optical vibrons in CdSe dots and dispersion relation of the bulk material. Phys. Rev. B, Vol. 57, No. 8, pp. 4664-4669.

Trinh, M. T.; Polak, L.; Schins, J. M.; Houtepen, A. J.; Vaxenburg, R.; Maikov, G. I.; Grinbom, G.; Midgett, A. G.; Luther, J. M.; Beard, M. C.; Nozik, A, J.; Bonn, M.; Lifshitz, E. \& Siebbeles, L. D. A., (2011). Anomalous Independence of Multiple Exciton Generation on Different Group IV-VI Quantum Dot Architectures. Nano Lett., Vol. 11, pp. 1623-1629. 
Underwood, D. F.; Kippeny, T. \& Rosenthal, S. J., (2001). Ultrafast carrier dynamics in CdSe nanocrystals determined by femtosecond fluorescence upconversion spectroscopy. J. Phys. Chem. B, Vol. 105, No. 2, pp. 436-443.

Valerini, D.; Cretì, A.; Lomascolo, M.; Manna, L.; Cingolani, R. \& Anni, M. Temperature dependence of the photoluminescence properties of colloidal CdSe/ZnS core/shell quantum dots embedded in a polystyrene matrix. Phys. Rev. B, Vol. 71, No. 23, pp. 235409-235414.

Velizhanin, K. A. \& Piryatinski, A., (2011). Numerical Study of Carrier Multiplication Pathways in Photoexcited Nanocrystal and Bulk Forms of PbSe. Phys. Rev. Lett., Vol. 106, pp. 207401-207404.

Wang, H. Y. ; de Mello Donegà, C.; Meijerink, A. \& Glasbeek, M., (2006). Ultrafast exciton dynamics in CdSe quantum dots studied from bleaching recovery and fluorescence transients. J. Phys. Chem. B, Vol. 110, No. 2, pp. 733-737.

Wang, X.; Qu, L.; Zhang, J.; Peng, X. \& Xiao, M. (2003a). Surface-Related Emission in Highly Luminescent CdSe Quantum Dots. Nano Lett., Vol. 3, No. 8, pp. 1103-1106.

Wang, X. Y.; Yu, W. W.; Zhang, J. Y.; Aldana, J.; Peng, X. \& Xiao, M., (2003b). Photoluminescence upconversion in colloidal CdTe quantum dots. Phys. Rev. B, Vol. 68, No. 12, pp. 125318-125323.

Woggon, U.; Giessen, H.; Gindele, F.; Wind, O.; Fluegel, B. \& Peyghambarian, N., (1996). Ultrafast energy relaxation in quantum dots. Phys. Rev. B, Vol. 54, No. 24, pp. 17681-17690.

Wolf, M.; Brendel, R.; Werner, J. H. \& Queisser, H. J., (1998). Solar cell efficiency and carrier multiplication in $\mathrm{Si}_{1-x} \mathrm{Ge}_{x}$ alloys. J. Appl. Phys., Vol. 83, No. 8, pp. 4213-4222.

Yoffe, A. D. (2001). Semiconductor quantum dots and related systems: electronic, optical, luminescence and related properties of low dimensional systems. Adv. Phys., Vol. 50, pp. 1-208.

Zhang, H.; Chen, D.; Zhang, J.; Wang, Z.; Cui, Y. \& Shen, L., (2010). Effect of Shell Layers on Luminescence of Colloidal CdSe $/ \mathrm{Zn}_{0.5} \mathrm{Cd}_{0.5} \mathrm{Se} / \mathrm{ZnSe} / \mathrm{ZnS}$ Core/Multishell Quantum Dots. Journal of nanoscience and nanotechnology, Vol. 10, No: 11, pp. 75877591.

Zhang, Y.; Miszta, K.; Kudera, S.; Manna, L.; Di Fabrizio, E. \& Krahne, R. (2011). Spatially resolved photoconductivity of thin films formed by colloidal octapod-shaped CdSe/CdS nanocrystals. Nanoscale, Vol. 3, No. 7, pp. 2964-2970.

Zheng, J. J.; Ji, W. Y.; Wang, X. Y.; Ikezawa, M.; Jing, P.; Liu, X.; Li, H.; Zhao, J. \& Masumoto, Y., (2010). Improved Photoluminescence of MnS/ZnS Core/Shell Nanocrystals by Controlling Diffusion of Mn Ions into the ZnS Shell. J. Phys. Chem. C, Vol. 114, No. 36, pp. 15331-15336. 


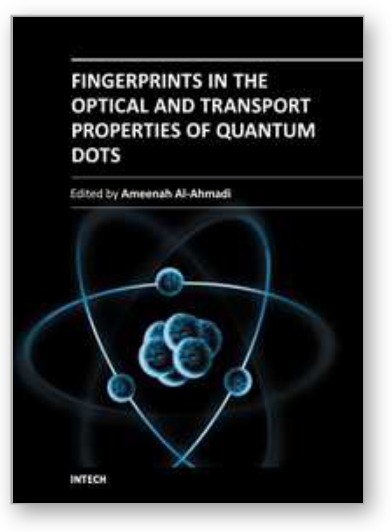

\author{
Fingerprints in the Optical and Transport Properties of Quantum \\ Dots \\ Edited by Dr. Ameenah Al-Ahmadi
}

ISBN 978-953-51-0648-7

Hard cover, 468 pages

Publisher InTech

Published online 13, June, 2012

Published in print edition June, 2012

The book "Fingerprints in the optical and transport properties of quantum dots" provides novel and efficient methods for the calculation and investigating of the optical and transport properties of quantum dot systems. This book is divided into two sections. In section 1 includes ten chapters where novel optical properties are discussed. In section 2 involve eight chapters that investigate and model the most important effects of transport and electronics properties of quantum dot systems This is a collaborative book sharing and providing fundamental research such as the one conducted in Physics, Chemistry, Material Science, with a base text that could serve as a reference in research by presenting up-to-date research work on the field of quantum dot systems.

\title{
How to reference
}

In order to correctly reference this scholarly work, feel free to copy and paste the following:

Giovanni Morello (2012). Optical Properties of Spherical Colloidal Nanocrystals, Fingerprints in the Optical and Transport Properties of Quantum Dots, Dr. Ameenah Al-Ahmadi (Ed.), ISBN: 978-953-51-0648-7, InTech, Available from: http://www.intechopen.com/books/fingerprints-in-the-optical-and-transport-properties-ofquantum-dots/optical-properties-of-spherical-colloidal-nanocrystals

\section{INTECH}

open science | open minds

\section{InTech Europe}

University Campus STeP Ri

Slavka Krautzeka 83/A

51000 Rijeka, Croatia

Phone: +385 (51) 770447

Fax: +385 (51) 686166

www.intechopen.com

\section{InTech China}

Unit 405, Office Block, Hotel Equatorial Shanghai

No.65, Yan An Road (West), Shanghai, 200040, China

中国上海市延安西路65号上海国际贵都大饭店办公楼 405 单元

Phone: +86-21-62489820

Fax: +86-21-62489821 
(C) 2012 The Author(s). Licensee IntechOpen. This is an open access article distributed under the terms of the Creative Commons Attribution 3.0 License, which permits unrestricted use, distribution, and reproduction in any medium, provided the original work is properly cited. 